\title{
Nonthermal phenomena in the center of Abell 1775
}

\section{An $800 \mathrm{kpc}$ head-tail, revived fossil plasma and slingshot radio halo}

\author{
A. Botteon ${ }^{1}$, S. Giacintucci ${ }^{2}$, F. Gastaldello ${ }^{3}$, T. Venturi ${ }^{4}$, G. Brunetti ${ }^{4}$, R. J. van Weeren ${ }^{1}$, T. W. Shimwell ${ }^{5,1}$, \\ M. Rossetti ${ }^{3}$, H. Akamatsu ${ }^{6}$, M. Brüggen ${ }^{7}$, R. Cassano ${ }^{4}$, V. Cuciti ${ }^{7}$, F. de Gasperin ${ }^{7}$, A. Drabent ${ }^{8}$, M. Hoeft ${ }^{8}$, \\ S. Mandal ${ }^{1}$, H. J. A. Röttgering ${ }^{1}$, and C. Tasse ${ }^{9,10}$
}

${ }^{1}$ Leiden Observatory, Leiden University, PO Box 9513, 2300, RA, Leiden, The Netherlands e-mail: botteon@strw.leidenuniv.nl

2 Naval Research Laboratory, 4555 Overlook Avenue SW, Code 7213, Washington, DC 20375, USA

3 INAF - IASF Milano, Via A. Corti 12, 20133 Milano, Italy

${ }^{4}$ INAF - IRA, Via P. Gobetti 101, 40129 Bologna, Italy

5 ASTRON, the Netherlands Institute for Radio Astronomy, Postbus 2, 7990, AA Dwingeloo, The Netherlands

6 SRON Netherlands Institute for Space Research, Sorbonnelaan 2, 3584, CA Utrecht, The Netherlands

7 Hamburger Sternwarte, Universität Hamburg, Gojenbergsweg 112, 21029 Hamburg, Germany

8 Thüringer Landessternwarte, Sternwarte 5, 07778 Tautenburg, Germany

9 GEPI, Observatoire de Paris, CNRS, Université Paris Diderot, 5 Place Jules Janssen, 92190 Meudon, France

10 Centre for Radio Astronomy Techniques and Technologies, Department of Physics and Electronics, Rhodes University, Grahamstown 6140, South Africa

Received 7 December 2020 / Accepted 2 March 2021

\begin{abstract}
Context. Thermal gas in the center of galaxy clusters can show substantial motions that generate surface-brightness and temperature discontinuities known as cold fronts. The motions may be triggered by minor or off-axis mergers that preserve the cool core of the system. The dynamics of the thermal gas can also generate radio emission from the intra-cluster medium (ICM) and impact the evolution of clusters' radio sources.

Aims. We aim to study the central region of Abell 1775, a system in an ambiguous dynamical state at $z=0.072$ which is known to host an extended head-tail radio galaxy, with the goal of investigating the connection between thermal and nonthermal components in its center.

Methods. We made use of a deep (100 ks) Chandra observation accompanied by LOFAR $144 \mathrm{MHz}$, GMRT $235 \mathrm{MHz}$ and $610 \mathrm{MHz}$, and VLA $1.4 \mathrm{GHz}$ radio data.

Results. We find a spiral-like pattern in the X-ray surface brightness that is mirrored in the temperature and pseudo-entropy maps. Additionally, we characterize an arc-shaped cold front in the ICM. We interpret these features in the context of a slingshot gas tail scenario. The structure of the head-tail radio galaxy "breaks" at the position of the cold front, showing an extension that is detected only at low frequencies, likely due to its steep and curved spectrum. We speculate that particle reacceleration is occurring in the outer region of this tail, which in total covers a projected size of $\sim 800 \mathrm{kpc}$. We also report the discovery of revived fossil plasma with ultra-steep spectrum radio emission in the cluster core together with a central diffuse radio source that is bounded by the arc-shaped cold front.

Conclusions. The results reported in this work demonstrate the interplay between thermal and nonthermal components in the cluster center and the presence of ongoing particle reacceleration in the ICM on different scales.
\end{abstract}

Key words. radiation mechanisms: non-thermal - radiation mechanisms: thermal - galaxies: clusters: general galaxies: clusters: intracluster medium - X-rays: galaxies: clusters - radio continuum: galaxies

\section{Introduction}

The intra-cluster medium (ICM) is a hot and rarified gas that permeates the entire cluster volume and has a temperature on the order of $10^{7}-10^{8} \mathrm{~K}$ and densities of about $10^{-3}-10^{-4}$ particles $\mathrm{cm}^{-3}$. In this regime, and for typical ICM metallicities $(0.1-1$ solar), the gas radiates $\mathrm{X}$-rays predominantly through thermal bremsstrahlung emission (e.g., Sarazin 1986, for a review). In the hierarchical process of large-scale structure formation,

\footnotetext{
* All reduced images are only available at the CDS via anonymous ftp to cdsarc.u-strasbg. fr (130.79.128.5) or via http://cdsarc. u-strasbg.fr/viz-bin/cat/J/A+A/649/A37
}

mergers between clusters leave observable imprints in the ICM in the form of shocks, cold fronts, and hydrodynamic instabilities (e.g., Markevitch \& Vikhlinin 2007, for a review). In minor and off-axis mergers, right after the pericenter passage, the gas tail of the infalling subcluster overshoots the dark matter peak due to the rapid decline of ram pressure and produces an arc-shaped slingshot tail (e.g., Hallman \& Markevitch 2004; Poole et al. 2006; Sheardown et al. 2019). The slingshot gas effect has been invoked in the context of gas sloshing in some of the more dynamically relaxed clusters, where the perturbations in the potential well of the more massive system induce a spiral-like motion of its cool core, which, without being disrupted, generates sloshing cold fronts in the ICM 
(Ascasibar \& Markevitch 2006). Several observations have provided evidence of these processes in the central regions of cool-core clusters (e.g., Churazov et al. 2003; Clarke et al. 2004; Laganá et al. 2010; Johnson et al. 2010, 2012; Blanton et al. 2011; Roediger et al. 2012; Sanders et al. 2016a; Chen et al. 2017; Ueda et al. 2017; Douglass et al. 2018), and the sloshing scenario is supported by a number of numerical simulations (e.g., Tittley \& Henriksen 2005; Ascasibar \& Markevitch 2006; ZuHone et al. 2010; Roediger et al. 2011).

The presence of turbulence in the cores of relaxed clusters is believed to play an important role in the formation of the diffuse synchrotron sources termed radio mini-halos (e.g., Gitti et al. 2002). Their steep spectrum ( $\alpha>1$, with $S_{v} \propto v^{-\alpha}$ where $\alpha$ is the spectral index) emission occupies the cluster cooling region $(r \sim 50-300 \mathrm{kpc})$ and is not directly caused by the active galactic nucleus (AGN) that sits in the central cluster galaxy (see Giacintucci et al. 2017, 2019, for a recent overview). Possible evidence for the connection between minihalos and sloshing motions is provided by observations showing the confinement of some mini-halos inside the region delineated by sloshing cold fronts (e.g., Mazzotta \& Giacintucci 2008; Hlavacek-Larrondo et al. 2013; Giacintucci et al. 2014a,b; Gendron-Marsolais et al. 2017). Indeed, gas sloshing has been suggested to amplify the magnetic field within the core region (e.g., Keshet \& Loeb 2010; ZuHone et al. 2011) and induce significant turbulent motions in the cluster center (Fujita et al. 2004; ZuHone et al. 2013), leading to the reacceleration of the relativistic electrons in mini-halos. Alternatively, it has been proposed that the radio-emitting electrons are generated by inelastic collisions between cosmic-ray protons and thermal protons (e.g., Pfrommer \& Enßlin 2004; Fujita et al. 2007; Zandanel et al. 2014); in this case, the confinement of the radio emission at cold fronts is expected to be less prominent (ZuHone et al. 2015).

The dynamics of the ICM can also impact the morphology and spectral properties of tailed cluster radio galaxies. Relativistic electrons in the tails can gain energy via a number of processes, such as turbulent reacceleration due to the growth of KelvinHelmholtz instabilities generated by the interaction between the radio plasma and the surrounding medium (e.g., Loken et al. 1995), adiabatic compression (e.g., Enßlin \& Krishna 2001), shock reacceleration (e.g., van Weeren et al. 2017), or even by more subtle mechanisms that barely balance electrons radiative losses powered by plasma instabilities within the radio tails (e.g., de Gasperin et al. 2017). Among these processes, adiabatic compression of pockets of old nonthermal components due to bulk motions in the ICM is believed to be responsible for the formation of radio emission with very steep spectra $(\alpha>2)$ called revived fossil plasma sources or radio phoenixes (e.g., Slee et al. 2001; Cohen \& Clarke 2011; van Weeren et al. 2011; Kale \& Dwarakanath 2012; Mandal et al. 2020).

The plethora of phenomena described above makes cluster centers unique laboratories to study the physics of diffuse, hot, weakly magnetized cosmic plasmas and their interplay with nonthermal components on a broad range of scales. The analysis of the central region of a cluster is indeed the focus of our study.

Abell 1775 is a galaxy cluster with a richness class of 2 belonging to the Boötes supercluster (Einasto et al. 1997; Chow-Martínez et al. 2014). Optical studies on the velocity distribution of cluster galaxies have revealed that it is a binary (Oegerle et al. 1995; Kopylov \& Kopylova 2009) or possibly ternary (Zhang et al. 2011) system. Here, we focus on its main component, Abell 1775B (hereafter simply A1775), located at $z=0.072$, which is known to host two giant ellip- tical galaxies at its center with a radial-velocity difference of $\sim 1800 \mathrm{~km} \mathrm{~s}^{-1}$ (Chincarini et al. 1971; Jenner 1974; Hintzen 1979; Kirshner et al. 1983; Zabludoff et al. 1990). Both galaxies are active in the radio band, with the southeast one, B1339+266B, whose radial-velocity deviates from the bulk of the velocities of the other cluster members, hosting an extended headtail radio source that has been studied at different frequencies and resolutions (Owen \& Ledlow 1997; Giovannini \& Feretti 2000; Giacintucci et al. 2007; Terni de Gregory et al. 2017). The dynamical state of the cluster is unclear: Lopes et al. (2018) classified A1775 as a relaxed system based on optical and X-ray information, Andersson et al. (2009) and Laganá et al. (2019) suggested the presence of dynamical motions in its center from spatially resolved X-ray spectral analysis, and the cool-core metrics used by Andrade-Santos et al. (2017) placed it in an ambiguous position between cool-core and non-cool-core systems. A1775 is part of the ROSAT Brightest Cluster Sample with an X-ray luminosity of $1.6 \times 10^{44} \mathrm{erg} \mathrm{s}^{-1}$ and a flux of $1.25 \times$ $10^{-11} \mathrm{erg} \mathrm{s}^{-1} \mathrm{~cm}^{-2}$ in the $0.1-2.4 \mathrm{keV}$ band (Ebeling et al. 1998). In the second catalog of Planck Sunyaev-Zel'dovich sources (Planck Collaboration XXVII 2016), it is reported with the name PSZ2 G031.93+78.71 and mass $M_{500}=(2.72 \pm 0.24) \times 10^{14} M_{\odot}$.

In this paper, we report on results based on deep Chandra observations and multifrequency radio observations performed with the LOw Frequency ARray (LOFAR), Giant Metrewave Radio Telescope (GMRT), and Very Large Array (VLA) spanning a frequency range from $144 \mathrm{MHz}$ to $1.4 \mathrm{GHz}$ with the aim of studying the central region of A1775 and the impact of the ICM dynamics on the diffuse radio sources in the cluster. We adopted a $\Lambda$ CDM cosmology with $\Omega_{\Lambda}=0.7$, $\Omega_{\mathrm{m}}=0.3$, and $H_{0}=70 \mathrm{~km} \mathrm{~s}^{-1} \mathrm{Mpc}^{-1}$, in which 1 arcsec corresponds to $1.372 \mathrm{kpc}$ at $z=0.072$. Unless otherwise stated, reported uncertainties correspond to a $68 \%$ confidence level.

\section{Data reduction}

\subsection{Chandra}

A1775 was observed twice (ObsIDs: 12891, 13510) with Chandra ACIS-S in VFAINT mode for a total exposure time of $100 \mathrm{ks}$. Observations were retrieved from the Chandra data archive and processed from the level $=1$ event file with CIAO v4.10 and using CALDB v4.7.8. We inspected the light curves extracted in the $0.5-7.0 \mathrm{keV}$ band from the S1 chip with the 1c_clean routine and did not find any periods affected by high background. The two ObsIDs were combined with merge_obs to produce an image in the $0.5-2.0 \mathrm{keV}$ band binned to have a pixel size of $0.984^{\prime \prime}$. Point sources were automatically detected with wavdetect, confirmed by visual inspection, and excluded in the subsequent analysis.

We obtained a background image by reprojecting the background templates normalized by counts in the $9.5-12 \mathrm{keV}$ band to the corresponding event files of the single ObsIDs. Surface-brightness analysis was performed with PROFFIT v1.5 (Eckert et al. 2011) on the exposure-corrected and backgroundsubtracted image of the cluster.

For each ObsID, spectra were extracted from the same regions with specextract and jointly fitted with XSPEC v12.10.0c (Arnaud 1996). The ICM emission was modeled assuming an absorbed thermal model (PHABS*APEC) with fixed redshift $(z=0.072)$ and column density $\left(N_{\mathrm{H}}=\right.$ $1.07 \times 10^{20} \mathrm{~cm}^{2}$; Willingale et al. 2013). Spectra were fit in the $0.5-7.0 \mathrm{keV}$ band adopting the solar abundance ratios from 
Asplund et al. (2009) and using normalized blank-sky observations to create background spectra. Maps of the thermodynamical quantities of the ICM were computed following Botteon et al. (2018) and employing CONTBIN v1.6 (Sanders 2006) to bin a $0.5-7.0 \mathrm{keV}$ band image adopting a signal-tonoise $(\mathrm{S} / \mathrm{N})$ of 50 for the net counts per spectral region.

\subsection{LOFAR}

A1775 falls within $2.5^{\circ}$ from the center of four pointings $(\mathrm{P} 207+25, \mathrm{P} 204+25, \mathrm{P} 207+27, \mathrm{P} 204+27)$ of the LOFAR Twometer Sky Survey (LoTSS; Shimwell et al. 2017, 2019). Each LoTSS pointing is observed with the LOFAR High Band Antenna (HBA) array operating at $120-168 \mathrm{MHz}$ and has an integration time of eight hours book-ended by ten-minute flux calibrator scans.

In the first step of the data reduction, the four observations were analyzed individually with the pipelines developed by the LOFAR Surveys Key Science Project team (PREFACTOR, van Weeren et al. 2016; Williams et al. 2016; de Gasperin et al. 2019; KILLMS, Tasse 2014a,b; Smirnov \& Tasse 2015; DDFACET, Tasse et al. 2018) to correct for direction-independent and dependent effects. In particular, we deployed the calibration pipeline ${ }^{1}$ v2.2, which includes improvements in terms of dynamic range, deconvolution of diffuse emission, image artifacts and image fidelity in general, delivering 6 " resolution images of the entire LOFAR field-ofview (FoV) with noise levels $\sim 100 \mu \mathrm{Jy}_{\text {beam }}{ }^{-1}$ (see Sect. 5 in Shimwell et al. 2019 and Tasse et al. 2021).

In the second step, we subtracted the sources outside a $33^{\prime} \times 33^{\prime}$ region that contains A1775 from the visibility data of each pointing by using the models derived from the pipeline. The extracted datasets are then phase-shifted to the center of the region, averaged, and corrected for the LOFAR station beam toward this direction. Residual artifacts were attenuated by means of phase and amplitude self-calibration loops on the combined measurement sets. This procedure is discussed in detail in van Weeren et al. (2020) and has already been adopted in several LOFAR HBA works (e.g., Hardcastle et al. 2019; Cassano et al. 2019; Botteon et al. 2019, 2020; Osinga et al. 2021; Hoang et al. 2021).

After the self-calibration step, the four observations are jointly imaged and deconvolved with WSCLEAN v2.8 (Offringa et al. 2014). Final images at the central observing frequency of $144 \mathrm{MHz}$ were obtained using the multiscale multifrequency deconvolution scheme (Offringa \& Smirnov 2017) with Briggs weightings (Briggs 1995) robust $=-1.25$ for the highest resolution image, or robust $=-0.5$ for lower resolution images.

It is known that the LOFAR flux density scale can show systematic offsets (e.g., Hardcastle et al. 2016). For this reason, we compared the flux density of the brightest compact sources in our new images with the mosaiced images from the forthcoming second LoTSS data release (Shimwell et al., in prep.). Therefore, we adopted a correction factor of 0.96 on LOFAR flux densities derived from the mean flux density ratio between our image and the LoTSS-DR2 mosaic. We set a conservative systematic uncertainty of $20 \%$ on LOFAR flux density measurements, as was done by LoTSS (Shimwell et al. 2019).

\footnotetext{
1 https://github.com/mhardcastle/ddf-pipeline
}

\subsection{GMRT}

We reanalyzed GMRT observations of A1775 at $235 \mathrm{MHz}$ and $610 \mathrm{MHz}$ that were previously published in Giacintucci et al. (2007). The cluster was observed in 2005 for $100 \mathrm{~min}$ at $235 \mathrm{MHz}$ and in 2003 for $45 \mathrm{~min}$ at $610 \mathrm{MHz}$ using the old hardware correlator and a bandwidth of $8 \mathrm{MHz}$ and $16 \mathrm{MHz}$, respectively. We refer the reader to Giacintucci et al. (2007) for further details on these observations.

We reprocessed the $235 \mathrm{MHz}$ data using the Astronomical Image Processing System (AIPS) starting from the raw visibilities to take advantage of the radio frequency interference (RFI) excision task RFLAG, which makes it possible to perform a careful flagging of bad data and was not available at the time of the previous analysis. We reduced the data following standard procedure, using 3C286 and 3C147 as primary calibrators to compute and apply gain and bandpass corrections to the data. 3C286 was also used to calibrate the data in phase. We used a combination of RFLAG and manual flagging on each source individually (calibrators and target) to remove RFI-affected visibilites and other bad data. We then appropriately averaged the calibrated target data to 21 frequency channels, each $250 \mathrm{KHz}$ wide, to reduce the dataset size while avoiding significant time or bandwidth smearing. We applied a number of phase-only self-calibration cycles to the target visibilities and used wide-field imaging by decomposing the primary beam area into $\sim 40$ smaller facets. We produced final images with the multi-scale CLEAN implemented in IMAGR. The rms sensitivity level achieved in the image at full resolution $\left(14^{\prime \prime} \times 10^{\prime \prime}\right.$, for ROBUST $=0$ in IMAGR) is $\sim 0.8 \mathrm{mJy} \mathrm{beam}^{-1}$, that is, $\sim 50 \%$ lower than the image noise in Giacintucci et al. (2007). We also produced images with lower resolution (down to $\sim 28^{\prime \prime}$ ) by increasing the ROBUST parameter and/or applying tapers to the $u v$ data during the imaging process. Overall, the combination of lower noise and better $u v$ coverage (resulting from the accurate RFI excision done with RFLAG) makes these new images more sensitive to faint and diffuse emission than that of Giacintucci et al. (2007).

We also attempted to recalibrate the $610 \mathrm{MHz}$ data presented in Giacintucci et al. (2007); however, no improvement was achieved compared the quality obtained in the previous work (we note thet RFI is less critical at this frequency than at $235 \mathrm{MHz}$ ). Therefore, we used the calibrated data at $610 \mathrm{MHz}$ from Giacintucci et al. (2007) to produce a new image at a resolution of $15^{\prime \prime}$ using the multi-scale CLEAN.

We corrected all images for the GMRT primary beam response $^{2}$ using PBCOR in AIPS. Residual amplitude errors are estimated to be within $15 \%$ at $235 \mathrm{MHz}$ and $10 \%$ at $610 \mathrm{MHz}$ (e.g., Chandra et al. 2004).

\subsection{VLA}

We obtained observations of A1775 at $1.4 \mathrm{GHz}$ from the VLA public archive. The cluster was observed in 2001 using the Darray configuration for a total of $1.4 \mathrm{~h}$ (project AL0515) and in 2004 for $2.5 \mathrm{~h}$ in C-configuration (project AV0265). We calibrated the data from the two configurations separately using AIPS and standard amplitude and phase calibration procedures. Bandpass calibration was applied to the D-configuration data that were taken using eight frequency channels in each of the two $25 \mathrm{MHz}$-wide intermediate frequency (IF) subbands. The flux density scale was set using the primary calibrators 3C147 and

\footnotetext{
2 http://www.ncra.tifr.res.in: 8081/ ngk/primarybeam/
} beam.html 


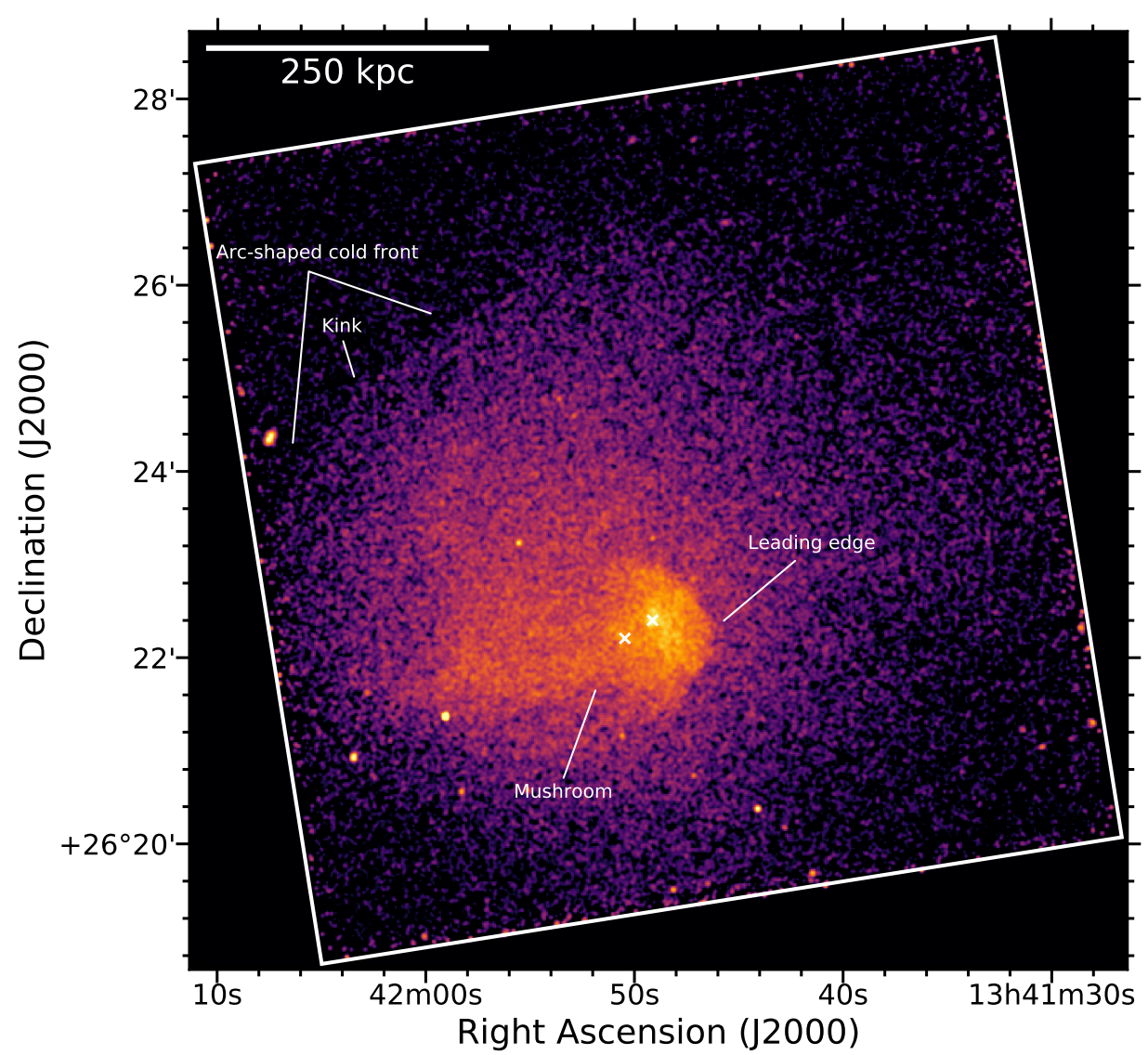

Fig. 1. Chandra X-ray image of A1775 in the $0.5-2.0 \mathrm{keV}$ band. The squared box region (for this and subsequent images) denotes the FoV of the ACIS-S3 chip. The main features discussed in the text are labeled in the figure. Crosses mark the position of the two giant elliptical galaxies.
3C286 and the Perley \& Butler (2017) coefficients in SET JY. We applied several loops of phase self-calibration to each dataset to reduce the effects of residual phase errors in the data. We then averaged the D-configuration visibilities to one single channel/IF and combined them with the C-configuration data in the $u v$ plane. Finally, we applied a final step of self-calibration in phase to the combined dataset. Residual flux calibration errors are estimated to be within $5 \%$.

\section{Results}

\subsection{X-ray arc-shaped feature}

The deep Chandra observation reveals an unusual system characterized by a bright core bounded by a sharp and prominent front in the W creating a mushroom-cap structure (Fig. 1). The bulk of the X-ray emission seems offset toward the NE, where a clear drop in surface brightness is also observed. To enhance the presence of these features, we produced the images shown in Fig. 2. On the left-hand panel, we report the residuals between the data and the best-fit spherical $\beta$-model ${ }^{3}$ (Cavaliere \& Fusco-Femiano 1976) centered on the image centroid (at RA: $13^{\mathrm{d}} 41^{\mathrm{m}} 51^{\mathrm{s}}$, Dec: $+26^{\circ} 23^{\prime} 00^{\prime \prime}$ ) that was adopted to describe the X-ray surface brightness (core radius $r_{\mathrm{c}}=2.56 \pm$ 0.05 arcmin and $\beta=0.84 \pm 0.01$ ). This image highlights a spiral pattern extending in clockwise direction from the cluster core. On the right-hand panel, we report the Gaussian gradient magnitude (GGM) image (Sanders et al. 2016b), which was obtained by combining images filtered on scales $\sigma_{\mathrm{GGM}}=[1,2,4,8,16]$ pixels with radial weighting. This representation highlights the

\footnotetext{
3 In Appendix A, we show that adopting an elliptical $\beta$-model does not
} lead to any appreciable difference. gradients of an image, such as surface-brightness discontinuities. For A1775 it emphasizes the leading edge ahead of the cluster core (the mushroom with a stem and a cap), as well as the same spiral noted in the residual image.

We investigated the thermodynamical properties of the cluster with temperature, pseudo-pressure, and pseudo-entropy maps of ICM that are produced in the way described in Sect. 2.1. These maps are shown in Fig. 3. In the mushroom, we detect a cool $(\sim 3 \mathrm{keV}) \mathrm{X}$-ray tail and a temperature jump at its W surfacebrightness front. The temperature is lower closer to the cluster center, indicating that we are observing a cold front. A detailed study of this edge will be presented in a forthcoming paper. Once again, a clear spiral pattern is observed in temperature and pseudo-entropy, where low-temperature and low-entropy gas is found to trace the overdense region of Fig. 2. In the NE direction, the CONTBIN algorithm found only one spectral region satisfying the requested $\mathrm{S} / \mathrm{N}$ because of lower count statistics due to the drop in surface brightness and greatest off-axis distance. A detailed analysis of this region is presented in the following.

At $\sim 500 \mathrm{kpc}$ from the cluster core, toward the NE, an arcsharped discontinuity can be seen by eye (Fig. 1). The feature is even clearer in the residual map and GGM-filtered image of Fig. 2, as well as by the spectral regions of Fig. 3 drawn by CONTBIN, which are optimized to follow the cluster X-ray brightness. We quantify the density jump in this direction by extracting and fitting X-ray surface-brightness profiles assuming spherical symmetry and an underlying a broken power-law density profile in the following form:

$$
\begin{array}{lll}
n_{\text {in }}(r)=C n_{0}\left(\frac{r}{r_{\mathrm{j}}}\right)^{a_{1}}, & \text { if } & r \leq r_{\mathrm{j}} \\
n_{\text {out }}(r)=n_{0}\left(\frac{r}{r_{\mathrm{j}}}\right)^{a_{2}}, & \text { if } & r>r_{\mathrm{j}}
\end{array},
$$



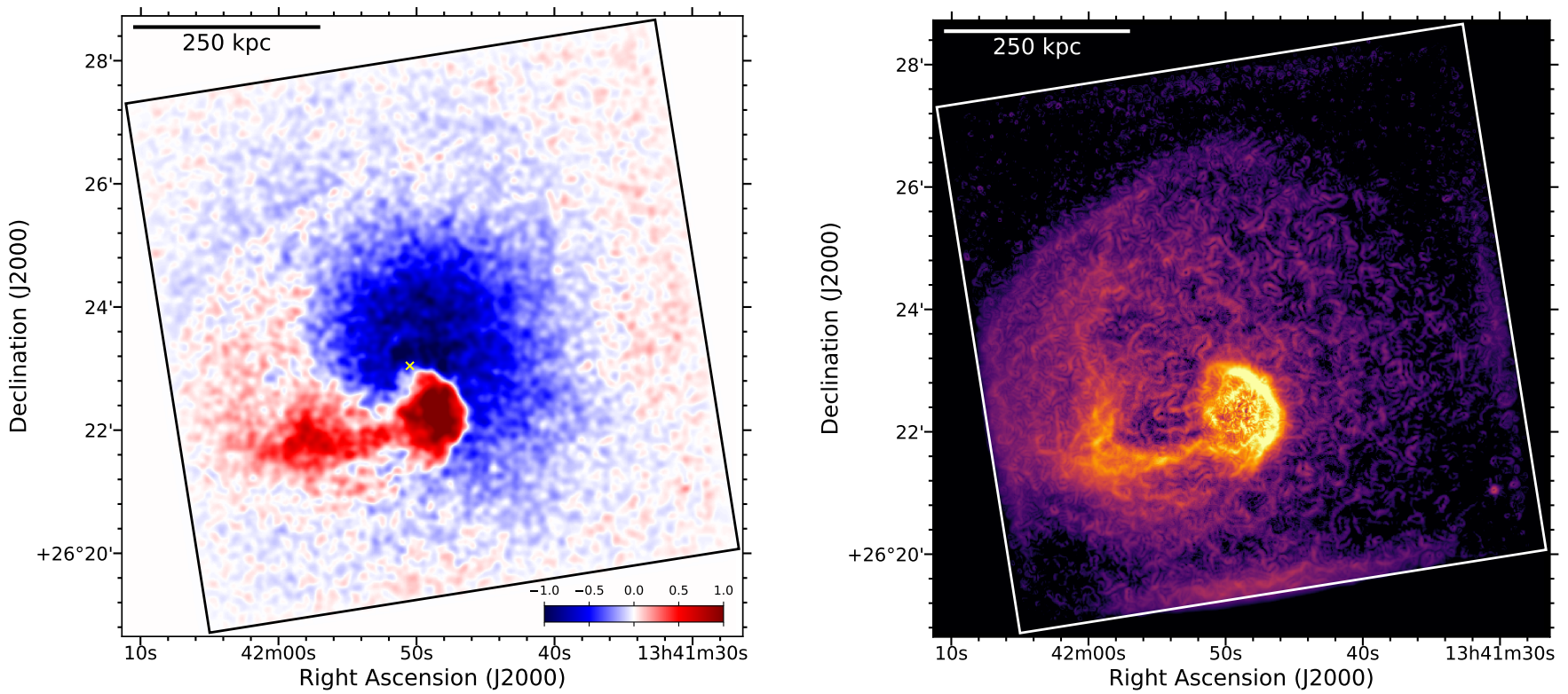

Fig. 2. Left: residuals (in units of $\sigma$ ) between the data and the best-fit $\beta$-model profile centered on the image centroid (marked by the cross). Right: GGM-filtered image with radial weighting.
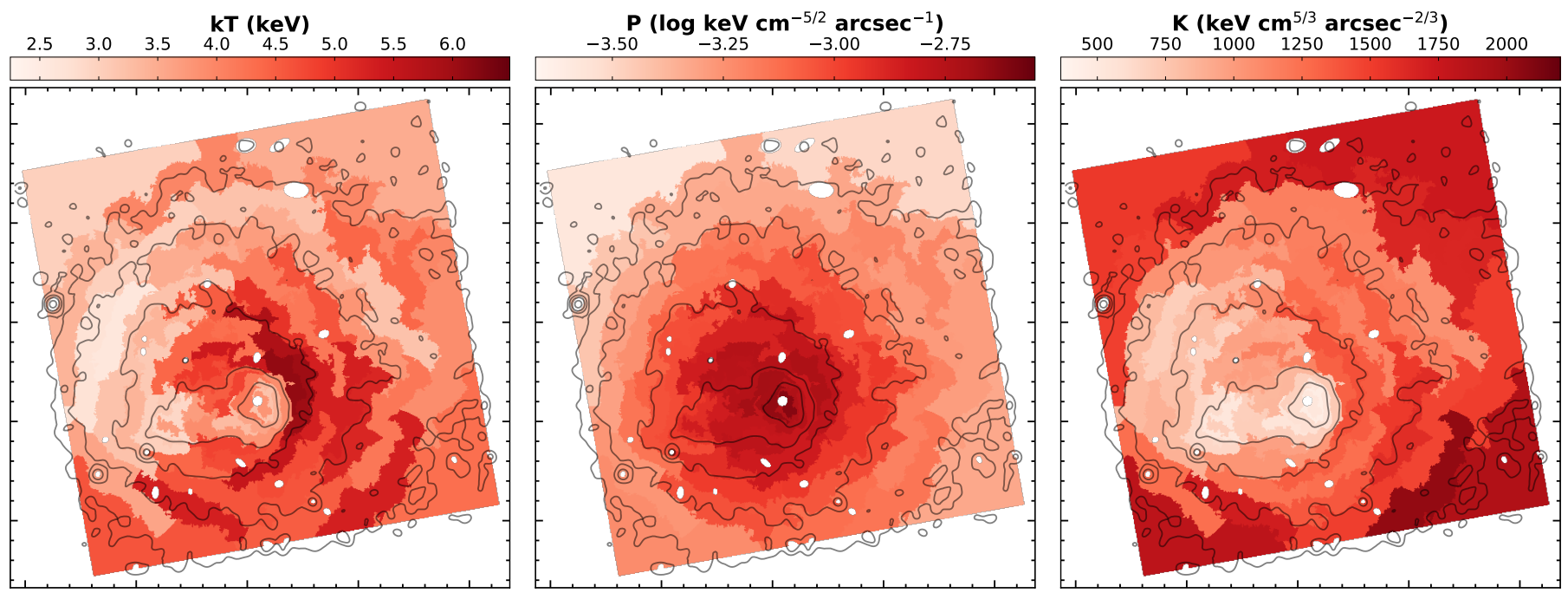

Fig. 3. Temperature (left), pseudo-pressure (center), and pseudo-entropy (right) maps with Chandra contours overlaid. The white ellipses mark the regions where point sources were removed. The typical fractional error in each region is in the range $\sim 5-10 \%$. The temperature error map is shown in Appendix B.

where $r$ denotes the radius from the center of the sector, $r_{\mathrm{j}}$ is the radius of the jump, $C \equiv n_{\text {in }} / n_{\text {out }}$ is the density ratio, $n_{\text {in }}$ and $n_{\text {out }}$ are the inner and outer densities, $n_{0}$ is a normalization factor, while $a_{1}$ and $a_{2}$ are the power-law indices. This choice is customarily adopted to model discontinuities in the ICM, such as shocks and cold fronts (e.g., Markevitch \& Vikhlinin 2007). The fitting was done leaving all the parameters free to vary. The analysis was performed in a "narrow" and a "wide" sector (centered at RA: $\left.13^{\mathrm{d}} 41^{\mathrm{m}} 51^{\mathrm{s}}, \mathrm{Dec}+26^{\circ} 22^{\prime} 24^{\prime \prime}\right)$. The former is entirely covered by the S3 chip and its angular aperture toward the $\mathrm{W}$ is extended up to the position of a surface-brightness kink. The latter covers the whole angular length of the discontinuity and extends beyond the chip FoV. The best-fit models and regions of the analysis are shown in Fig. 4. We find that the broken-power law model provides a good description of the data. The density ratio (deprojected along the line of sight under the assumption of spherical geometry by PROFFIT) and radial distance of the discontinuity are consistent between the two regions: $C=1.67_{-0.10}^{+0.11}$ and $r_{\mathrm{j}}=3.75_{-0.03}^{+0.02}$ arcmin (narrow sector) and $C=1.73_{-0.10}^{+0.11}$ and $r_{\mathrm{j}}=3.79_{-0.02}^{+0.03}$ arcmin (wide sector). The best-fit radius of the jump is slightly offset in the wide sector due to the presence of a kink in the discontinuity (at RA: $13^{\mathrm{h}} 42^{\mathrm{m}} 03^{\mathrm{s}}$, Dec: $+26^{\circ} 24^{\prime} 57^{\prime \prime}$, see Fig. 1), possibly suggesting that its curvature radius mildly changes across the front.

Spectral analysis across the discontinuity was performed in five regions ( 3 inwards and 2 outwards) obtained from the narrow sector of Fig. 4, which avoids the edge of the S3 chip. In order to take into account the contribution of each annular shell into the neighboring ones, we made use of the PROJCT model in XSPEC to deproject the data in 3D under the assumption of spherical symmetry. In Fig. 5, we report the projected and deprojected radial temperature profiles. Although the large uncertainties do 

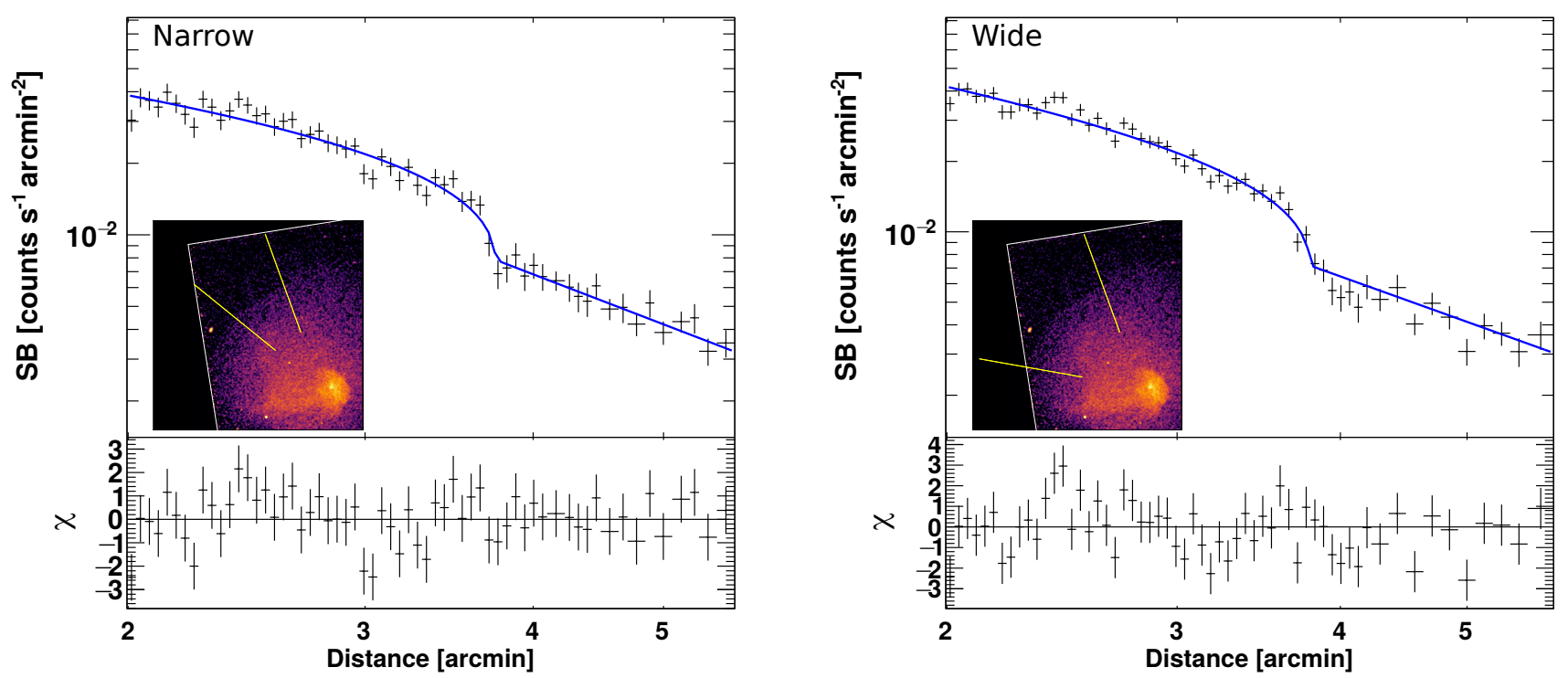

Fig. 4. Surface-brightness profiles in the $0.5-2.0 \mathrm{keV}$ band and best-fit broken power-law models for the NE discontinuity. Inset panels: sectors used for the analysis. The $\chi^{2}$ dd.o.f. of the fits are $65.31 / 56$ (narrow sector) and 73.99/53 (wide sector).

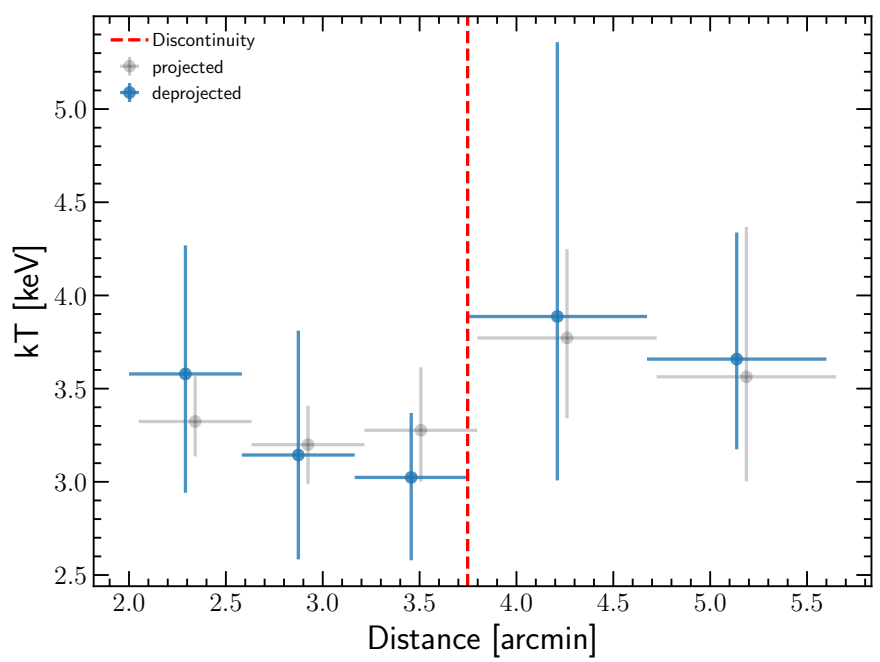

Fig. 5. Deprojected (with PROJCT) temperature profile across the narrow sector of Fig. 4. The position of the surface-brightness discontinuity is marked by the dashed line. The projected profile is slightly offset in the $x$-direction for clarity.

not allow us to claim a sharp temperature jump, we find that the internal region is characterized by lower temperature values, as observed in the case of cold fronts. In the two spectral sectors just inwards and outwards of the surface-brightness edge, we measure $k T_{\text {in }}=3.0_{-0.4}^{+0.3} \mathrm{keV}$ and $k T_{\text {out }}=3.9_{-0.9}^{+1.5} \mathrm{keV}$, respectively. The observation of an arc-shaped cold front accompanied by a spiral pattern in the residual and gradient images and temperature and pseudo-entropy maps, indicate the presence of substantial gas motions in the central region of A1775. The dynamical state of the cluster and the origin of the arc-shaped feature are discussed in Sect. 4.1.

\subsection{Head-tail radio galaxy}

The most prominent radio sources in A1775 are the extended head-tail radio galaxy $(\mathrm{B} 1339+266 \mathrm{~B})$ and the double radio source $(B 1339+266 A)$, which originate from two elliptical galaxies of similar luminosity, separated by a projected distance of $32 \mathrm{kpc}$ (Parma et al. 1991). The association between the radio emission and the host galaxy was done by Giacintucci et al. (2007). In Fig. 6, we show our LOFAR $144 \mathrm{MHz}$ image of A1775 at a resolution of $5^{\prime \prime} \times 3^{\prime \prime}$ overlaid with an optical image from the Panoramic Survey Telescope and Rapid Response System (Pan-STARRS; Chambers et al. 2016). Both sources are detected at high $\mathrm{S} / \mathrm{N}$, and some residual artifacts due to ionospheric phase corruptions can be noticed around the two elliptical galaxies. The radio structures in the eastern direction are discussed in the next section.

A multifrequency view of the radio emission from A1775 at $144 \mathrm{MHz}$ (LOFAR), $235 \mathrm{MHz}$ (GMRT), $610 \mathrm{MHz}$ (GMRT), and $1.4 \mathrm{GHz}$ (VLA) is shown in Fig. 7. The GMRT and VLA images are shown at lower resolution to enhance the sensitivity to extended emission, which is better recovered in the LOFAR image despite the higher resolution. The emission of the head-tail radio galaxy can roughly be divided into three components: the bright head, the previously reported emission of the tail extending for $\sim 400 \mathrm{kpc}$, and a newly discovered lowsurface-brightness extension that is only observed at $144 \mathrm{MHz}$ and $235 \mathrm{MHz}$ (Fig. 7). The latter component is diffuse and originates after a "break" of the collimated tail. The newly discovered diffuse emission from the tail extends toward the NE for an additional $\sim 400 \mathrm{kpc}$ and is not detected at higher frequencies, likely due to its steep spectrum. Overall, the head-tail radio galaxy in A1775 spans $\sim 800 \mathrm{kpc}$ (projected), making it one of the longest tailed sources observed in galaxy clusters to date (see e.g., Abell 1132; Wilber et al. 2018 and Abell 2256; Owers et al. 2014 for two noteworthy examples). From our LOFAR highresolution image that is shown in Fig. 6, we measure a total flux density of $S_{144}=2.8 \pm 0.6 \mathrm{Jy}$ for the tailed source (uncertainties are dominated by the $20 \%$ calibration error). This flux density is distributed among the three components of the headtail radio galaxy as follows: $1.2 \mathrm{Jy}$ for the head, $1.3 \mathrm{Jy}$ for the "inner" $400 \mathrm{kpc}$-tail, and 0.3 Jy for the "outer" tail extension. In Fig. 8, we show the values of the tail together with the GMRT and VLA flux densities, that are also summarized in Table 1 together with the other radio sources in A1775. Since the outer 


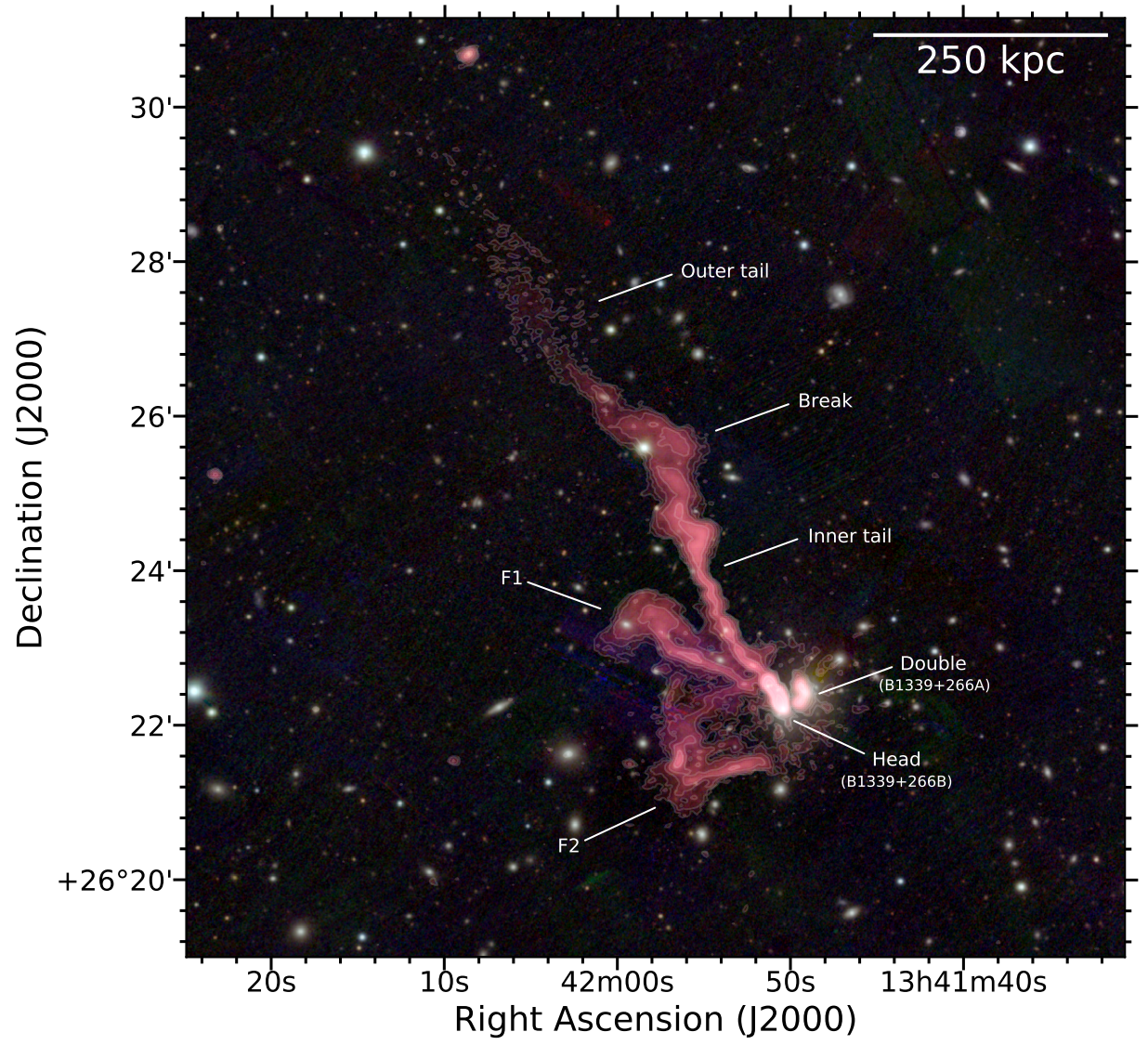

Fig. 6. Composite optical-radio image obtained from Pan-STARRS $g, r, i$ and LOFAR $144 \mathrm{MHz}$ high-resolution $\left(5^{\prime \prime} \times 3^{\prime \prime}\right)$ data. Radio contours start from $3 \sigma$, where $\sigma=148 \mu \mathrm{Jy}$ beam $^{-1}$, and they are spaced by a factor of 2 . The main features discussed in the text are labeled in the figure. tail is not detected at $610 \mathrm{MHz}$ and $1.4 \mathrm{GHz}$, we report the $1 \sigma$ upper limits on the emission. The inner tail follows a power-law with $\alpha_{144}^{610}=1.06 \pm 0.02$ between $144 \mathrm{MHz}$ and $610 \mathrm{MHz}$ (the $235 \mathrm{MHz}$ data is included in this estimate), then it steepens to $\alpha_{610}^{1400}=1.69 \pm 0.14$ at higher frequencies, suggesting spectral curvature. The outer tail spectral index is not well constrained between $144 \mathrm{MHz}$ and $235 \mathrm{MHz}$ due to the narrow frequency span, implying $\alpha_{144}^{235}=1.23 \pm 0.52$. We note that this range of values is not consistent with the upper limit at $1.4 \mathrm{GHz}$ if the spectrum were a power law. This indicates that the spectrum of this component is curved.

We used the GMRT $235 \mathrm{MHz}$ and VLA $1.4 \mathrm{GHz}$ observations, together with the LOFAR $144 \mathrm{MHz}$ dataset, to create low(144-235 MHz) and high- (144-1400 MHz) frequency spectral index images. To measure the same spatial scales, we applied the inner and outer $u v$ ranges of GMRT and VLA observations to our LOFAR dataset to create the images used to produce the $144-235 \mathrm{MHz}$ and the $144-1400 \mathrm{MHz}$ spectral index maps. These ranges were $70 \lambda$ to $23 k \lambda$ and $160 \lambda$ to $16.2 k \lambda$ for the GMRT and VLA, respectively. The images at each frequency band were convolved to the same resolution $\left(28^{\prime \prime} \times 28^{\prime \prime}\right)$, corrected for positional offsets, and regridded to have an identical pixelation before combination. These spectral index maps, shown in Fig. 9, are useful to study the aging of relativistic electrons in synchrotron emitting sources. In particular, the 144-1400 MHz map, thanks to its broad coverage that spans one decade in frequency, allows us to provide good constraints on the spectral index of the radiation. However, it is insensitive to the steep spectrum emission detected only at low frequencies. In this respect, the $144-235 \mathrm{MHz}$ map can give indications about the presence of the steepest spectrum emission, with the caveat that the precise value of $\alpha$ can be affected by the uncertainties intro- duced by the narrow frequency span. We note that both the maps in Fig. 9 show typical values in the core of $\alpha=0.6-0.7$, and indicate spectral steepening along the tail. As mentioned before, the slightly lower (and noiser) values of the $144-235 \mathrm{MHz}$ spectral index map are likely related to the shorter frequency pivot between the two observations and to the spectral curvature at higher frequencies. In Sect. 4.3, we discuss the surface brightness and spectral features observed along the head-tail radio galaxy in detail.

\subsection{Revived fossil plasma and central diffuse radio emission}

Bright, irregular, diffuse radio emission is observed to the $\mathrm{E}$ of the two dominant elliptical galaxies of A1775 (Fig. 6). Two main high-surface-brightness filaments can be identified in the LOFAR image: F1 (accounting for $593 \pm 119 \mathrm{mJy}$ ) is close to the brightest region of the head-tail radio galaxy, while F2 (accounting for $505 \pm 101 \mathrm{mJy}$ ) is located in the southern region and elongated in the E-W direction. Diffuse emission is observed between these two structures, and this has a total flux density of $\simeq 150 \mathrm{mJy}$ (this value is more uncertain due to residual artifacts in the image). This complex emission covers an area of $\sim 230^{2} \mathrm{kpc}^{2}$ in projection, is detected in the LOFAR $144 \mathrm{MHz}$ and GMRT $235 \mathrm{MHz}$ images (but is well recovered only by the former), and not at higher frequencies (Fig. 7). This suggests that it has a steep spectrum. Indeed, the distribution of the pixel values in the 144-235 MHz spectral index map (Fig. 9) for the two regions that cover the two main filaments have a consistent median of $\bar{\alpha}=2.4$ (with standard deviation $\sigma_{\alpha}=0.4$ ). This is in agreement with the non-detections at $610 \mathrm{MHz}$ and $1.4 \mathrm{GHz}$ and generally indicates that the emission has an ultra-steep spectrum $(\alpha>2)$, whose precise value can only be determined with 


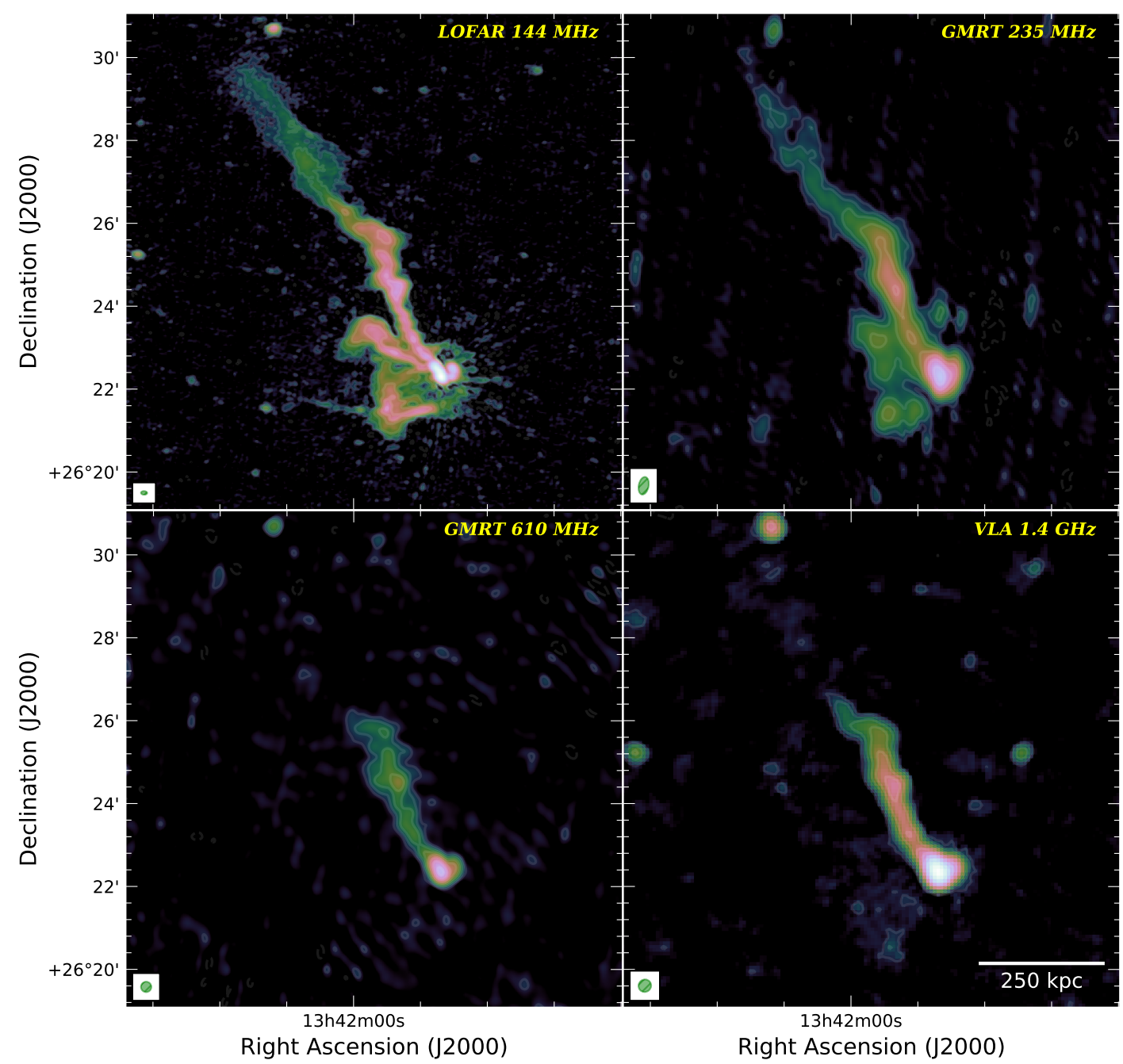

Fig. 7. LOFAR $144 \mathrm{MHz}\left(9^{\prime \prime} \times 5^{\prime \prime}\right)$, GMRT $235 \mathrm{MHz}\left(26^{\prime \prime} \times 14^{\prime \prime}\right)$, GMRT $610 \mathrm{MHz}\left(15^{\prime \prime} \times 15^{\prime \prime}\right)$, and VLA C+D array $1.4 \mathrm{GHz}\left(19^{\prime \prime} \times 18^{\prime \prime}\right)$ images. Contours are spaced by a factor of 2 starting from $3 \sigma$, where $\sigma_{144}=105 \mu \mathrm{Jy} \mathrm{beam}^{-1}, \sigma_{235}=0.9 \mathrm{mJy} \mathrm{beam}^{-1}, \sigma_{610}=680 \mu \mathrm{Jy} \mathrm{beam}^{-1}$, and $\sigma_{1400}=66 \mu \mathrm{Jy}_{\text {beam }^{-1}}$. The color scale has a logarithmic stretch from 1 to $3000 \sigma$. Beams are shown in the bottom left corners.

future deeper observation at $\gtrsim 300 \mathrm{MHz}$. Given the morphology of the radio source, its very steep spectrum, the lack of clear optical counterpart(s), and the possible co-location with some compression regions in the ICM (see end of Sect. 4.2), we conclude that we are observing revived fossil plasma emission. An obvious candidate for the origin of these filaments is the double giant elliptical system in the core, that in the past may have injected the relativistic plasma in the ICM that has been subsequently revived by the gas dynamics triggered by motions in the core.

When the longest baselines of the LOFAR observation are tapered down, further low-surface-brightness diffuse radio emission with roundish morphology is detected in the cluster center (Fig. 10). Although this newly discovered emission is strongly contaminated by the much brighter head-tail radio galaxy and the revived fossil plasma source, it is not obviously connected to any of them. This fainter emission is extended towards the $\mathrm{NE}$, where it is remarkably confined by the arc-shaped cold front detected with Chandra (Sect. 3.1), reported with the dashed yellow arc in Fig. 10.

The flux density of the central diffuse radio emission, excluding the contamination of sources, is in the range of 70-80 mJy (depending on the adopted extracting region). It is likely that

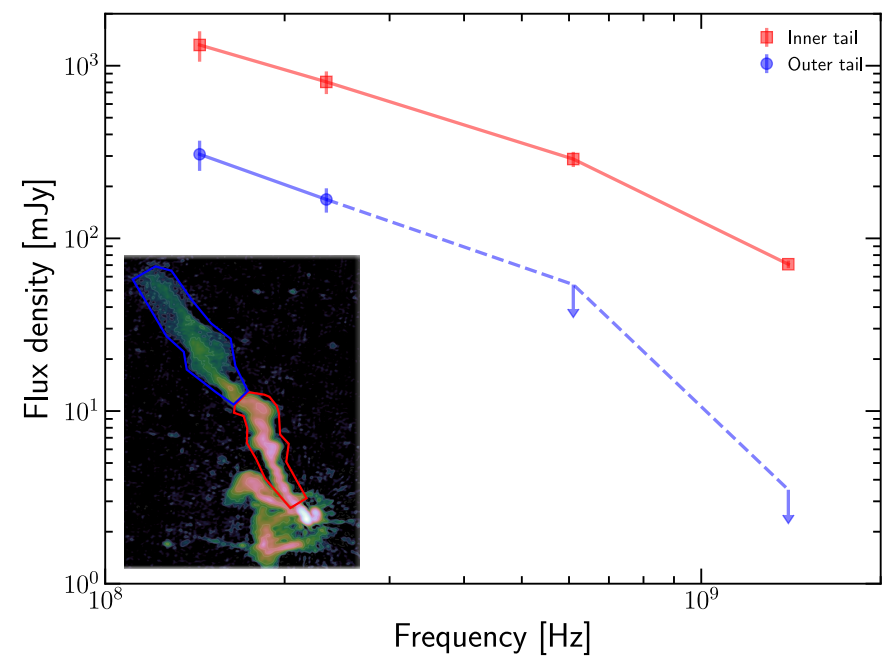

Fig. 8. Integrated spectra of the "inner" and "outer" regions (shown in the inset panel) of the head-tail radio galaxy.

this diffuse emission also extends up to the regions where the emission of the head-tail radio galaxy and revived fossil plasma 
Table 1. Flux densities of the radio sources in A1775.

\begin{tabular}{|c|c|c|}
\hline Source & $v[\mathrm{MHz}]$ & $S_{v}[\mathrm{mJy}]$ \\
\hline Double & 144 & $2402 \pm 480$ \\
\hline \multirow[t]{2}{*}{ Head } & 144 & $1213 \pm 243$ \\
\hline & 144 & $1319 \pm 264$ \\
\hline \multirow{3}{*}{ Inner tail } & 235 & $806 \pm 121$ \\
\hline & 610 & $288 \pm 29$ \\
\hline & 1400 & $701 \pm 3.6$ \\
\hline \multirow{4}{*}{ Outer tail } & 144 & $307 \pm 61$ \\
\hline & 235 & $168 \pm 27$ \\
\hline & 610 & $<54$ \\
\hline & 1400 & $<3.5$ \\
\hline $\mathrm{F} 1$ & 144 & $593 \pm 119$ \\
\hline $\mathrm{F} 2$ & 144 & $505 \pm 101$ \\
\hline Central diffuse emission & 144 & $244 \pm 50$ \\
\hline
\end{tabular}

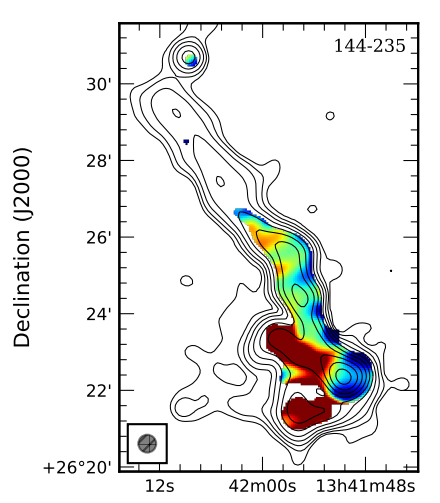

Right Ascension (J2000)

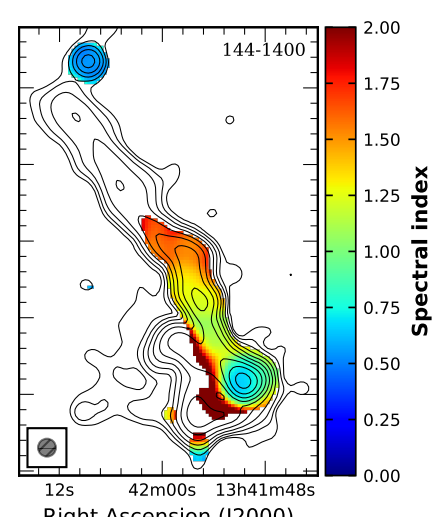

Right Ascension (J2000)
Fig. 9. Low (144-235 MHz) and high (144-1400 MHz) frequency spectral index maps at a resolution of $28^{\prime \prime} \times 28^{\prime \prime}$ with LOFAR contours at the same resolution overlaid. The beam is shown in the bottom left corner. Pixels with values below 5 and $3 \sigma$ were blanked in the lowand high-frequency spectral index maps, respectively. The error maps are shown in Appendix B.

source is dominating. One could attempt to estimate the total flux density assuming that the central diffuse emission follows an exponential profile (e.g., Murgia et al. 2009) in the following form:

$$
I(r)=I_{0} \mathrm{e}^{-r / r_{e}}
$$

where $I_{0}$ is the central surface brightness and $r_{e}$ denotes the $e$ folding radius, and employing the recently developed Halo-Flux Density CAlculator ${ }^{4}$ (HALO-FDCA; Boxelaar et al. 2021). This code allows us to estimate the flux density of extended diffuse sources such as giant and mini-halos in galaxy clusters. It performs a fit of the surface-brightness profile of the emission in 2D, assuming exponential profiles, allowing for regions of the image affected by contaminating sources to be masked and extrapolating the flux density beneath them. The fitting follows a Markov chain Monte Carlo (MCMC) method to explore the parameter space. After masking the contribution of the sources embedded in the central diffuse emission of A1775, we performed the fit with HALO-FDCA assuming the circular model of Eq. (2). The low-resolution image used for the fit with the adopted masks, together with the best-fit model and residual image, are shown in Fig. 11. The best-fit values are $I_{0}=16.2 \pm 1.1 \mu \mathrm{Jy} \operatorname{arcsec}^{-2}$

\footnotetext{
4 https://github.com/JortBox/Halo-FDCA
}

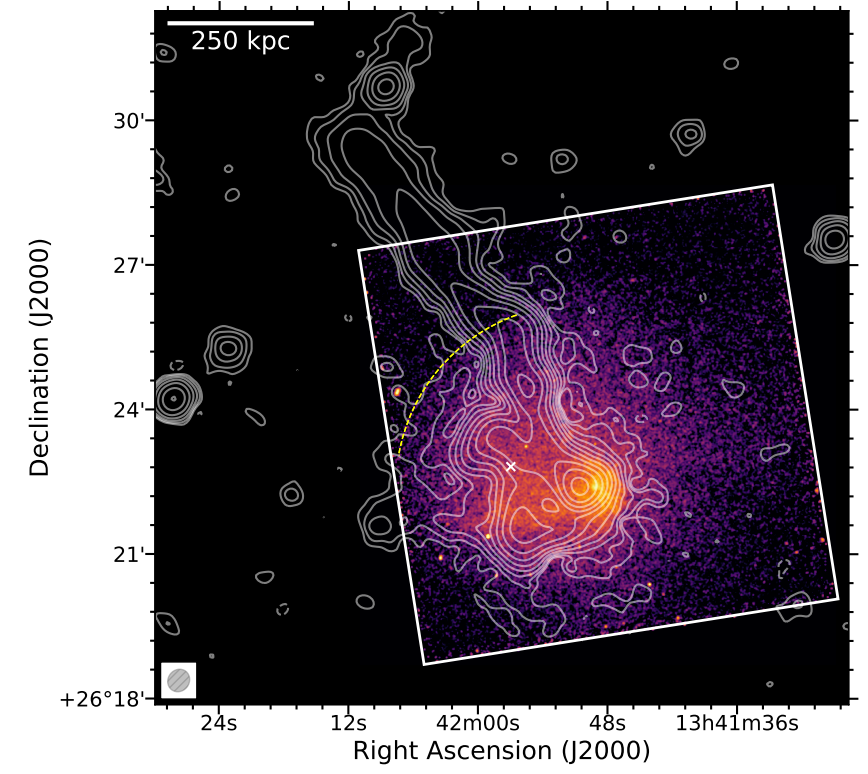

Fig. 10. Low-resolution $\left(29^{\prime \prime} \times 26^{\prime \prime}\right)$ LOFAR contours overlaid on the Chandra image. Contours are spaced by a factor of 2 starting from $3 \sigma$, where $\sigma=255 \mu \mathrm{Jy}_{\text {beam }}{ }^{-1}$. The beam is shown in the bottom left corner. The dashed yellow arc marks the position of the cold front traced by the X-ray surface-brightness discontinuity of Fig. 4 while the white cross denotes the center of the circular model shown in Fig. 11.

and $r_{e}=75 \pm 2 \mathrm{kpc}$. The total flux density obtained integrating the profile up to $3 r_{e}$ is $S_{144}=245 \pm 50 \mathrm{mJy}$. We stress that the majority of the diffuse flux is masked out, and the derived flux density relies on the validity of the extrapolation of the model in the masked regions, which cannot be tested. The measured flux density leads to a radio power of $P_{144}=4 \pi D_{\mathrm{L}}^{2} S_{144}=$ $(3.1 \pm 0.7) \times 10^{24} \mathrm{~W} \mathrm{~Hz}^{-1}$ (we neglected the $k$-correction term due to the low $z$ of the cluster and the unknown spectral index). The properties observed (i.e., source size, confinement of the emission in the cold front, and radio power) may indicate that the central diffuse emission is a radio mini-halo. However, as we see in Sect. 4.1, the classic definition of a mini-halo is challenged by the possible dynamical state of the cluster, and therefore in Sect. 4.2 we propose an alternative classification of the radio emission.

\section{Discussion}

\subsection{Dynamical state of the cluster and origin of the arc-shaped feature}

Literature studies do not agree on the dynamical state of A1775 (Andersson et al. 2009; Andrade-Santos et al. 2017; Lopes et al. 2018; Laganá et al. 2019). Although the cluster hosts a core that is colder than the surrounding medium (Fig. 3), it cannot be unambiguously defined as a cool-core or relaxed system. Andrade-Santos et al. (2017) used four metrics to identify coolcore clusters in a large sample of galaxy clusters observed with Chandra, and found that the values of A1775 (Table 2) are intermediate between cool-core and non-cool-core systems. Additionally, taking $3 \mathrm{keV}$ as central temperature (Fig. 3) and using the central density reported in Andrade-Santos et al. (2017), we estimate a central entropy $K_{0}=k T n_{\text {core }}^{-2 / 3} \simeq 52 \mathrm{keV} \mathrm{cm}^{2}$, which is again close to the usual threshold adopted to separate the two classes of clusters (e.g., Cavagnolo et al. 2009). The detailed view of the cluster central region provided by the new Chandra 

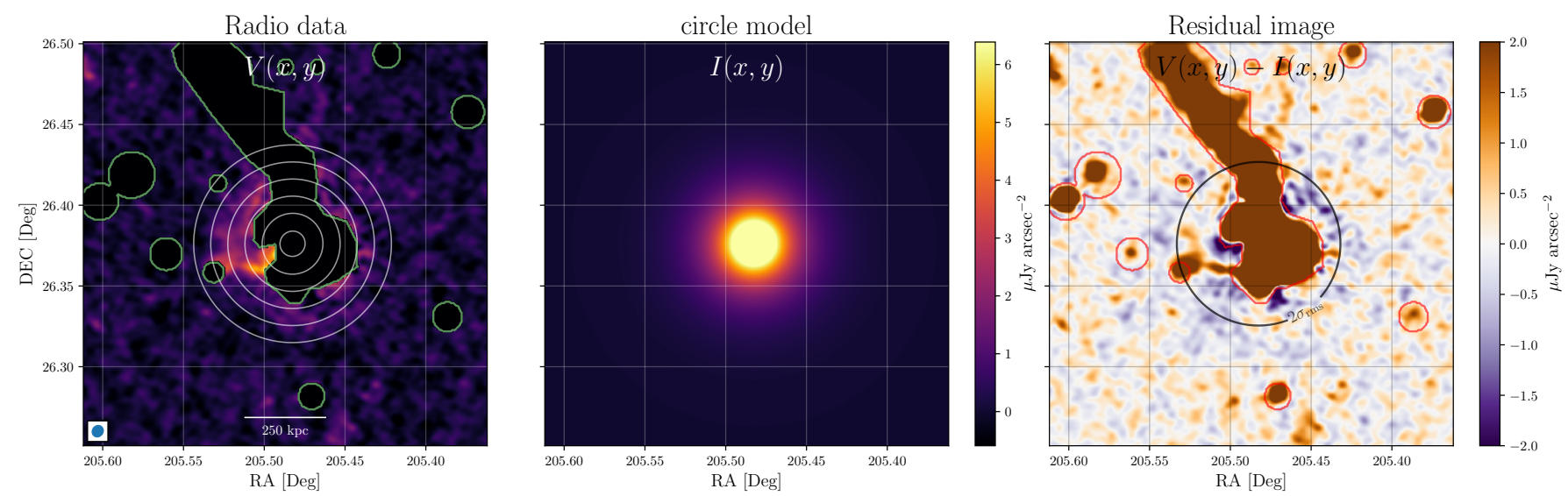

Fig. 11. Fitting result obtained with HALO-FDCA (Boxelaar et al. 2021). Left: LOFAR low-resolution image used for the fit. Contaminating regions are masked out and contoured in green. The circular contours show the model at $[1,2,4, \ldots, 32] \times \sigma$ levels. Center: image of the best-fit circular model. Right: residual image of the fit. The circle shows the $2 \sigma$ level of the model. The red contours shows the masked regions. The $\chi^{2} /$ d.o.f. of the fit is $1422.35 / 828$. The MCMC corner plot of the fit is reported in Appendix B.

Table 2. Concentration parameters $\left(C_{\mathrm{SB}}\right.$ and $\left.C_{\mathrm{SB} 4}\right)$, cuspiness $\left(\delta_{\text {cusp }}\right)$, and central gas density $\left(n_{\text {core }}\right)$ derived by Andrade-Santos et al. (2017) for A1775.

\begin{tabular}{lcc}
\hline \hline Parameter & Value & Definition used \\
\hline$C_{\mathrm{SB}}$ & $0.279 \pm 0.005$ & Maughan et al. (2012) \\
$C_{\mathrm{SB} 4}$ & $0.0823 \pm 0.0021$ & Santos et al. (2008) \\
$n_{\text {core }}\left[\mathrm{cm}^{-3}\right]$ & $0.01393 \pm 0.00065$ & - \\
\hline
\end{tabular}

Notes. Cool-core clusters typically have $C_{\mathrm{SB}}>0.4, C_{\mathrm{SB} 4}>0.075$, $\delta_{\text {cusp }}>0.5$, and $n_{\text {core }}>0.015 \mathrm{~cm}^{-3}$.

observation allowed us to highlight the presence of gas motions and X-ray discontinuities in the ICM (Sect. 3.1). In view of our analysis, we offer two possible scenarios to explain the observed properties of A1775, hence its dynamical state.

In a "sloshing scenario", when a galaxy cluster is perturbed, its cool core starts sloshing in the cluster's potential well, generating a spiral of low-entropy, low-temperature gas, which is separated by the hot ICM by contact discontinuities (e.g., Ascasibar \& Markevitch 2006). In A1775, we observe a spiral-pattern in the X-ray residual image of the best-fit surfacebrightness profile, in the GGM-filtered image, and in the temperature and pseudo-entropy maps. The central diffuse radio emission confined by the arc-shaped cold front (Fig. 10) could then be interpreted as a radio mini-halo bounded by a sloshing cold front. We note that A1775 is part of a supercluster, indicating that it is in a dynamically active environment. This scenario could explain some of the properties of A1775 (i.e., the spiral of low-entropy gas and the arc-shaped cold front) and has also been advanced by Laganá et al. (2019). Nonetheless, it cannot explain all the aspects of the system. In the classical sloshing scenario proposed by Ascasibar \& Markevitch (2006), the cluster core should remain largely intact (i.e., compact and peaked in the X-rays), while it oscillates in the potential well. The core at the center of A1775 seems to be in a disruption phase and has a leading surface-brightness edge on the W (Fig. 1) that is reminiscent of a "remnant-core" cold front, rather than a sloshing cold front (see Tittley \& Henriksen 2005; Markevitch \& Vikhlinin 2007; ZuHone \& Roediger 2016 for reviews). Furthermore, the presence of two elliptical galaxies with similar luminosity and high radial-velocity difference (Chincarini et al. 1971; Jenner 1974) is difficult to reconcile with the scenario of a relaxed cluster with a sloshing core.

In a "slingshot scenario", low-entropy or low-temperature gas spirals and arc-shaped features in the ICM can trace slingshot tails produced by off-axis mergers (e.g., Sheardown et al. 2019). The presence of a semi-disrupted core at the center of A1775 together with the two elliptical galaxies with a radial-velocity difference of $\sim 1800 \mathrm{~km} \mathrm{~s}^{-1}$ (Chincarini et al. 1971; Jenner 1974) possibly suggest that A1775 underwent a more violent merger compared to that required for the sloshing scenario. In this case, the core that we are observing at the center of A1775 is that of a subcluster that fell into the system along the line of sight and with a nonzero impact parameter. The subcluster is now close to the apocenter of its orbit, producing a slingshot tail, and it is turning around for the second infall generating the leading edge on the $\mathrm{W}$. The fact that there is only one core in A1775 can be explained if the main system did not host a cool core before the collision with the subcluster. Overall, the proposed scenario explains the features observed but requires a particular merger configuration and viewing angle.

Spiral-like structures and arc-shaped cold fronts in the ICM are typically generated by off-axis mergers. The collision with nonzero impact parameter is one of the conditions that generates sloshing motions and slingshot tails, which produce similar features and sometimes can be confused (see Sect. 7 in Sheardown et al. 2019). At the moment, for A1775 we tend to prefer the slingshot scenario because: (i) the cluster core is not compact as expected in the case of "classic" sloshing", (ii) there is an X-ray discontinuity in the $\mathrm{W}$ that has the characteristics of a remnant-core cold front, and (iii) the cluster center has two elliptical galaxies with a strong velocity component along the line of sight.

X-ray spectral analysis can also provide information on the bulk motions of the ICM and therefore further constraints on the dynamical state of the system. However, the limited spectral resolution of CCDs makes this measurement really

5 Here, we refer to the scenario of Ascasibar \& Markevitch (2006), where a necessary condition for the onset of the sloshing is the presence of a steep entropy profile. We note, however, that sloshing has also been proposed in the literature for a few clusters without a cool core and with indications of merging activity, such as Abell 2142 (Rossetti et al. 2013), Abell 3560 (Venturi et al. 2013), and Abell 1763 (Douglass et al. 2018). 

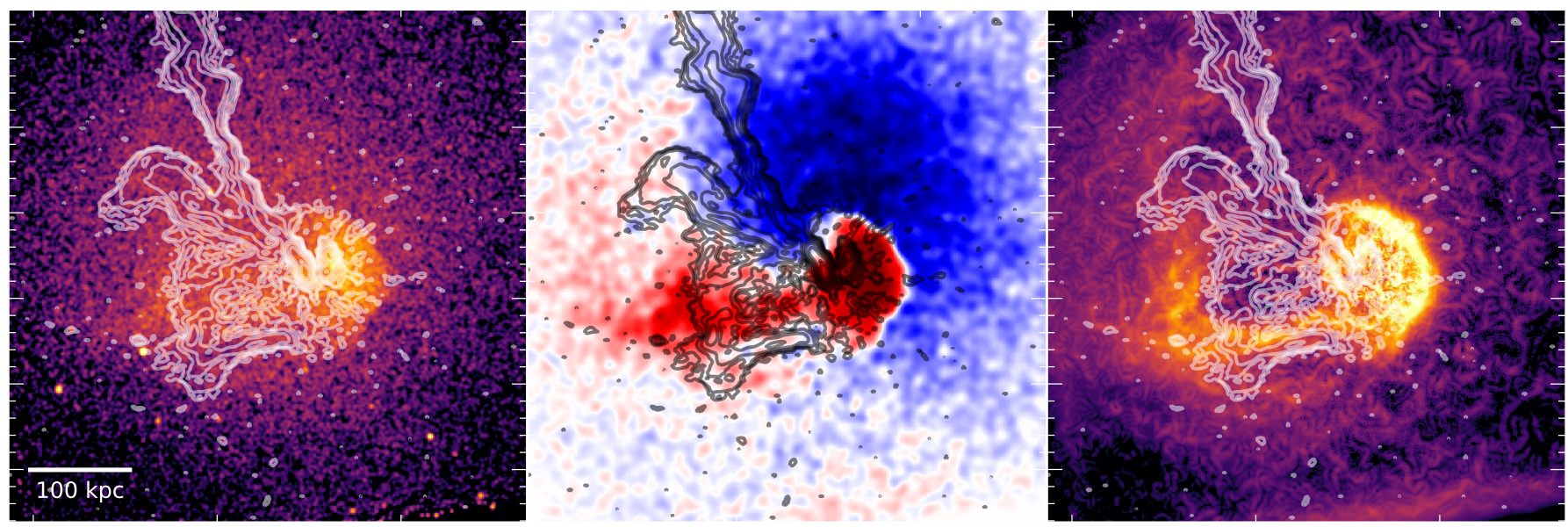

Fig. 12. Zoom-in on the central cluster region. From left to right: the LOFAR high-resolution contours of Fig. 6 are overlaid on the Chandra X-ray image, residual map, and GGM-filtered image of Figs. 1 and 2.

challenging and plagued by systematics. In the past, some detections, generally associated with large uncertainties, were claimed with Chandra (e.g., Dupke \& Bregman 2001, 2006; Dupke et al. 2007). More recently, Liu et al. (2015, 2016) proposed a strategy to infer bulk motions in clusters mainly with ACIS-I data once again recognizing, however, the difficulty of taking these measurements. In particular, the characterization of the time and spatial gain variation, such as that done with $X M M$ Newton in the recent work by Sanders et al. (2020), is crucial to performing the search for bulk motions. This type of analysis is very difficult to perform with ACIS-S and is beyond the scope of this paper. With all these caveats in mind, we attempted to measure the redshift from X-ray spectral analysis in different ICM regions but we were able to obtain reliable results only for the head of the mushroom (in a circular region with $r=40^{\prime \prime}$ ), where the fit provided $z=0.0719_{-0.007}^{+0.003}$. This value lies in the middle of the redshifts of the two elliptical galaxies $\left(z_{\mathrm{B} 1339+266 \mathrm{~A}}=0.0757 \pm 0.0001\right.$ and $z_{\mathrm{B} 1339+266 \mathrm{~B}}=$ $0.0693 \pm 0.0001$, Davoust \& Considere 1995) and is possibly in line with the scenario of a merger along the line of sight. Outside the mushroom, the redshifts derived from the X-rays are basically unconstrained. In the future, the high-spectral resolution provided by microcalorimeters on-board upcoming X-ray missions such as XRISM (XRISM Science Team 2020) and Athena X-IFU (Nandra et al. 2013) will enable this kind of science.

The detailed analysis of the dynamical state of the cluster, the identification of optical/X-ray substructures, and the analysis of the main cold front in the $\mathrm{W}$ will be the subject of subsequent papers.

\subsection{Connection between gas motions and central diffuse radio emission: a slingshot radio halo?}

The determination of the dynamical state of a cluster is important to understand the origin of the extended synchrotron sources located in cluster centers, namely giant radio halos and radio mini-halos. While the former have a diffuse emission that generally extends over Mpc scales and are found in merging clusters, the latter cover the cooling region of relaxed systems and typically surround the AGN of the central cluster galaxy (e.g., van Weeren et al. 2019, for a review). Relativistic electrons in giant and mini-halos are believed to gain energy via mergerinduced or sloshing-induced turbulent reacceleration processes, respectively (e.g., Brunetti \& Jones 2014, for a review). So, how does the central diffuse radio emission discovered in A1775 compare to this context?

On the one hand, the radio emission may resemble that of a mini-halo. First, it is located at the center of a cluster that has a distinct core with lower temperature or entropy than the ambient medium (Fig. 3). Second, it has an $e$-folding radius of $75 \pm 2 \mathrm{kpc}$, while giant halos in the sample of Murgia et al. (2009) generally have $r_{e}>100 \mathrm{kpc}$ (at $1.4 \mathrm{GHz}$ ). Third, it is confined by an arc-shaped cold front in the NE (Fig. 10), as is often observed in sloshing and mini-halo systems. On the other hand, mini-halos have only been found in systems with $K_{0}<30 \mathrm{keV} \mathrm{cm}^{2}$ (Giacintucci et al. 2017) so far, while the central entropy of A1775 is $K_{0} \simeq 52 \mathrm{keV} \mathrm{cm}^{2}$. Moreover, in Sect. 4.1 we argue that central gas sloshing, usually invoked to explain the origin of radio mini-halos, is not the preferred dynamical scenario for A1775. The small linear extent and relative high radio power, when compared to the low-mass of the system, point against the classification as giant radio halo (however, we note that to date giant radio halos have mainly been studied in massive systems undergoing major mergers, and their properties in low-mass clusters are still largely unconstrained). All this suggests that the extended emission should be classified in another way.

Pursuing the discussion on the cluster dynamical state (Sect. 4.1), we introduce and adopt the term "slingshot radio halo" for the central diffuse emission in A1775. The synchrotron emission appears mini-halo-like because sloshing and the slingshot develop under the same condition and produce similar features. It follows that sloshing spirals and slingshot tails are governed by the same physics. The difference between a mini-halo and a slingshot radio halo would then only be the dynamics that induced the gas motions in the ICM.

According to the turbulent reacceleration models, synchrotron-emitting electrons in mini-halos originate from a pre-existing population of seed relativistic electrons that are injected in the ICM by cluster AGN and that are spread and may be reaccelerated over $\lesssim 500 \mathrm{kpc}$ by sloshing-induced turbulent motions (e.g., ZuHone et al. 2013). The direct observations of these motions is very challenging, and, at the moment, constraints on the gas velocity dispersion are available only for the Perseus cluster, indicating that its ICM is sloshing at the level of a few hundred $\mathrm{km} \mathrm{s}^{-1}$ (Hitomi Collaboration 2016; Sanders et al. 2020). In slingshot tails, the atmosphere of the infalling system can be efficiently stripped near pericenter 

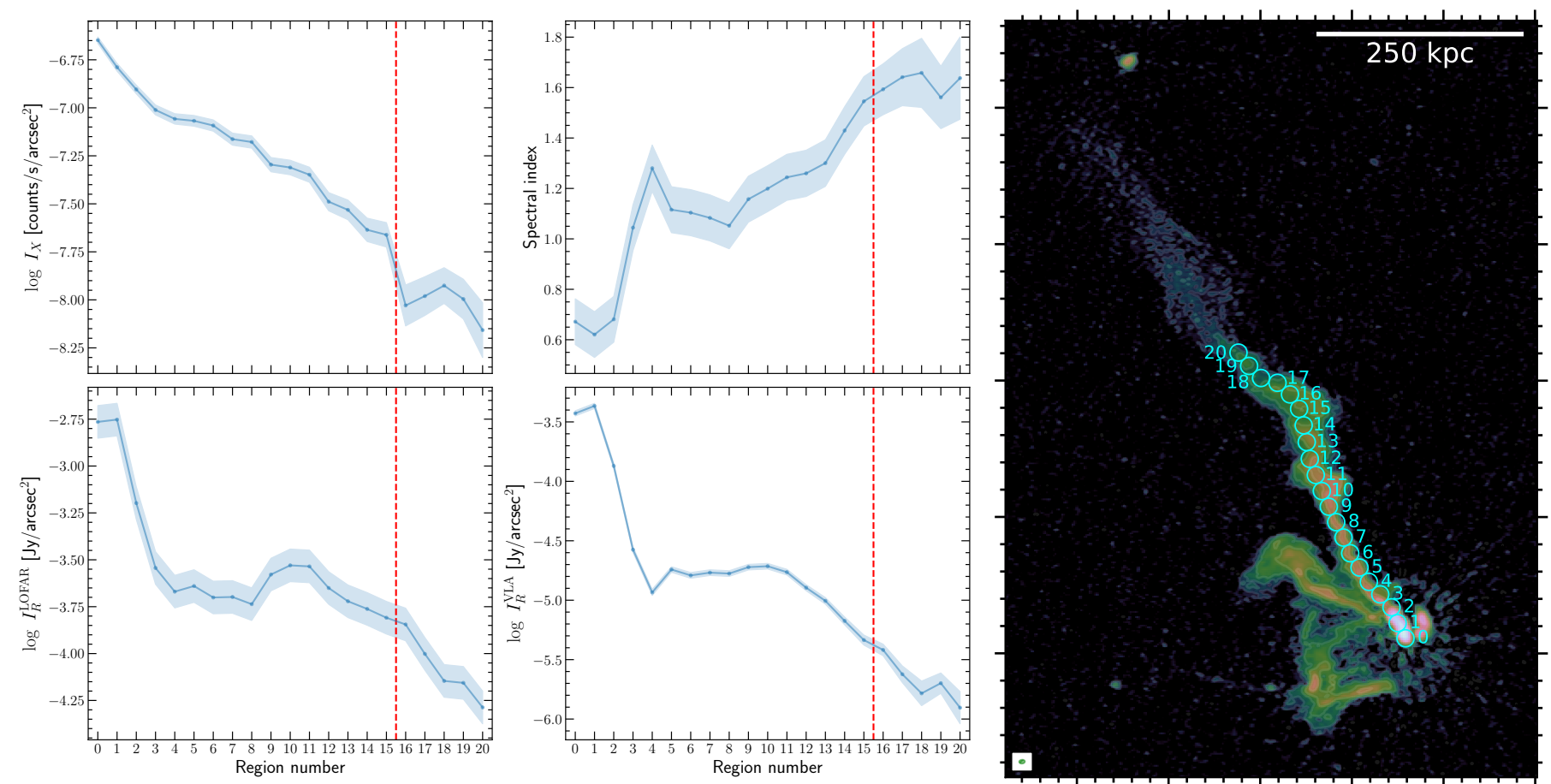

Fig. 13. Spectral index, LOFAR, and VLA radio surface brightness $\left(I_{\mathrm{R}}\right)$, and Chandra X-ray surface-brightness $\left(I_{\mathrm{X}}\right)$ trends along the head-tail radio galaxy. Radio surface brightnesses and spectral indexes were computed from LOFAR and VLA C-array images with a beam of $15^{\prime \prime} \times 15^{\prime \prime}$. Measurements were performed in the cyan beam-sized circular regions reported in the right panel, which depicts the same LOFAR high-resolution image of Fig. 6 for visualization purposes only (the beam is shown in the bottom left corner). Regions are numbered from 0 (head of the tail) to 20 (last region where the VLA emission is $>3 \sigma$ ). The red dashed vertical line on the left panels denotes the location of the arc-shaped cold front.

passage and become turbulent (e.g., Roediger et al. 2015), leading to the reacceleration of relativistic electrons via collisionless damping of turbulence. Arc-shaped sloshing or slingshot cold fronts are instead easier to observe and their origin is well understood. Beneath these discontinuities, shear magnetic amplification can increase the field strength (Keshet \& Loeb 2010; ZuHone et al. 2011; Reiss \& Keshet 2014), leading to the stabilization of the fronts against Kelvin-Helmholtz instabilities. In turn, relativistic plasma can be restrained by this magnetic field and form diffuse radio emission bounded by $\mathrm{X}$-ray edges. In A1775, the confinement of the slingshot radio halo within the discontinuity is remarkable (Fig. 10), and it is well in line with the scenario described above.

Seed relativistic electrons are a key ingredient of turbulent re-acceleration models as the direct acceleration of thermal pool electrons is a very inefficient mechanism. Cluster radio galaxies are a natural source of particles in the ICM. Interestingly, two radio loud elliptical galaxies are found at the center of A1775. One of the two is a giant head-tail radio galaxy that spans a projected size of about $800 \mathrm{kpc}$, meaning that it has the potential to inject relativistic electrons on a very large scale. In addition, we reported the presence of revived fossil plasma embedded in the diffuse radio emission which is direct evidence for the existence of seed particles in the environment. Therefore, the requirement of a pre-existing population of cosmic-ray electrons is clearly satisfied in this cluster.

The images shown in Fig. 12 show a tantalizing connection between the radio emissions $\mathrm{F} 1$ and F2 and some features observed in the X-rays. In particular, F1 follows the ridge between the overdense and underdense region in the Chandra residual image (central panel) while F2 is coincident with a region where a strong $\mathrm{X}$-ray surface-brightness gradient is present (right panel). It is possible that these old radio (light) filamentary structures passively trace the velocity field of the turbulent medium that is established by the interplay and mixing of the cold gas of the infalling subcluster with that of the main system.

The distortions observed on a linear scale $\sim 70 \mathrm{kpc}$ in $\mathrm{F} 1$ and F2 may result from shear motions in the velocity field that eventually generate a turbulent cascade in the ICM. These distortions suggest that the medium in the slingshot tail is quite turbulent with a velocity of the large-scale eddies that may be estimated as $\delta V_{1 \mathrm{D}} \sim L / t_{\text {cross }}$ where $L \sim 70 \mathrm{kpc}$ and $t_{\text {cross }}$ is the crossing time of the cold gas (CG), $t_{\text {cross }} \sim D / V_{\mathrm{CG}}, D \sim 200 \mathrm{kpc}$ being the (projected) distance between the front of the leading edge (the cap of the mushroom) and the region where the distortions of F1 and F2 are developed. The resulting turbulent Mach number on about $70 \mathrm{kpc}$ scale assuming isotropic turbulence and sound speed $c_{\mathrm{s}} \sim 1000 \mathrm{~km} \mathrm{~s}^{-1}$ is $\mathcal{M} \sim 0.3\left(\frac{V_{\mathrm{CG}}}{500 \mathrm{~km} \mathrm{~s}^{-1}}\right)$. These conditions open to the possibility of turbulent acceleration with acceleration times of a few $100 \mathrm{Myr}$, which are not very different from those assumed in giant radio halos and radio bridges (e.g., Brunetti \& Lazarian 2016; Brunetti \& Vazza 2020) and may explain the existence of steep spectrum diffuse radio emission on a scale of several hundreds of $\mathrm{kpc}$.

\subsection{Extended head-tail}

Our low-frequency observations with LOFAR and GMRT indicate that the head-tail radio galaxy is twice the size of the one previously reported (Fig. 7), leading to a projected largest linear size of $\sim 800 \mathrm{kpc}$. Interestingly, the newly discovered $\sim 400 \mathrm{kpc}$ extension discovered has different properties compared to the 
first part of the tail. It originates after a "break" of its structure, becoming more diffuse and wider, and shows an approximately constant surface brightness at $144 \mathrm{MHz}$ (i.e., close to the break frequency suggested by Fig. 7, assuming that adiabatic losses and strong variations of magnetic field do not play an important role), possibly indicating that the oldest population of electrons has been disturbed and reenergized, similarly to the case of the head-tail radio galaxies in Abell 1132 (Wilber et al. 2018) and $\mathrm{ZwCl} 0634.1+4750$ (Cuciti et al. 2018, and in prep.). The number of tailed radio galaxies showing this kind of feature is increasing thanks to the advent of highly sensitive observations at low frequencies, which are also possibly unveiling new gentle mechanisms of particle reenergization in tailed sources, as has been proposed for the case of the wide-angle radio galaxy in Abell 1033 (de Gasperin et al. 2017). As shown in Fig. 10, the tail breaks roughly at the location of the cold front detected in the X-rays. This suggests an interaction between the tail and the surrounding medium. Although the current data do not allow us to perform an accurate and detailed analysis of the spectral properties of the tail, the integrated spectra hint at the presence of a steep and curved spectrum for the newly discovered extension of the tail.

The analysis presented in Sect. 3.2 highlights the presence of a gradient along the tail, from $\alpha \sim 0.6-0.7$ up to $\alpha \gtrsim$ 1.5 , which is in agreement with that previously reported by Giacintucci et al. (2007). In order to study the trends observed along the source structure in more detail, we produced the plots reported in Fig. 13. There, we compare the Chandra, LOFAR, and VLA surface brightnesses, and the LOFAR-VLA spectral index between $144 \mathrm{MHz}$ and $1.4 \mathrm{GHz}$ measured in the same 21 circular regions depicted in the right-hand panel following the ridge line of the head-tail where the emission is $>3 \sigma$ in all observations. In order to increase the number of (beam-sized) regions for the analysis, we made use of a higher resolution VLA C-array image, and the final analysis was done on images convolved to the same resolution of $15^{\prime \prime} \times 15^{\prime \prime}$. Below, we briefly comment each panel of Fig. 13.

The Chandra surface brightness $\left(I_{\mathrm{X}}\right)$ declines toward the end of the tail. This behavior is expected because the head of the tail is located in the core of the cluster, that is the X-ray brightest region of the ICM. Between regions 15 and 16, the surface brightness drops rapidly due to the presence of the cold front (see also Fig. 4).

In the LOFAR surface-brightness profile $\left(I_{\mathrm{R}}^{\mathrm{LOFAR}}\right)$, the two innermost regions correspond to the head of the tail, where the radio surface brightness is highest. Then, the emission sharply decreases until it reaches region 4 , and it remains roughly constant for five regions. Between regions 8 and 9 , the tail gets brighter, and it fades again after region 11 . Once the tail has crossed the X-ray discontinuity, its emission seems to decline faster.

The VLA surface brightness $\left(I_{\mathrm{R}}^{\mathrm{VLA}}\right)$ shows an initial trend similar to that observed in the LOFAR image, with the difference that the surface brightness gets brighter between regions 4 and 5 before remaining roughly constant until region 11 (a small enhancement is noted between region 8 and 11, where the LOFAR brightness shows a more pronounced "bump"). Afterwards, the emission decreases regularly until it reaches the final region.

The spectral index has a fast steepening from $\alpha \sim 0.6-0.7$ in the inner regions up to $\alpha \sim 1.3$ in region 4 . The spectral index then gets flatter $(\alpha \sim 1.1)$ before steepening again reaching values up to $\alpha \sim 1.6$ in the last region. Within the uncertainties (shaded area), the steepening is consistently grad- ual along the tail. The investigation of possible variations in the spectral index trend (suggested by the mean values measured, such as between region 8 and 11) requires follow-up observations.

Once a relativistic jet has interacted with the ambient medium, it decelerates due to the entrainment of the external thermal gas (e.g., Bicknell 1984; Laing \& Bridle 2002a,b) and flares due to the nonlinear growth of Kelvin-Helmholtz instabilities (e.g., Jones \& Owen 1979; Loken et al. 1995). The position of the flaring point depends on how efficiently the interstellar medium of the host galaxy is stripped due to ram pressure during its transonic motion in the ICM (Gunn \& Gott 1972). In the samples of tailed sources presented by O'Donoghue et al. (1993) and Sebastian et al. (2017), the location of the flaring point varies from a minimum of $\sim 15 \mathrm{kpc}$, reaching values up to $\sim 100 \mathrm{kpc}$. These values may be affected by projection effects. For the head-tail in A1775, we found that the radio surface brightness shows two possible flaring points: between regions 4 and 5 at $1.4 \mathrm{GHz}$ (where also a jump in spectral index index is present), and between regions 8 and 9 at $144 \mathrm{MHz}$ (Fig. 13), corresponding to projected distances from region 0 of $\sim 103 \mathrm{kpc}$ and $\sim 185 \mathrm{kpc}$, respectively. At such large distances, radio flaring can also be induced by dynamical motions in the ICM. The interplay between the head-tail radio galaxy and the thermal gas is evident where the structure of the tail breaks and changes direction. This transition occurs at the position of the arc-shaped cold font (cf. Fig. 10), where both a tangential gas velocity variation and a density gradient are present. The disruption and bending of the tail is expected in this case, where the development of Kelvin-Helmholtz instabilities can in principle reaccelerate the electrons and sustain their particle life-time (e.g., Loken et al. 1995). Follow-up observations will shed light on the properties of the extension of the radio tail and test this scenario. We note that the possible interaction between a radio galaxy and a cold front has been recently claimed for NGC 4869 in the Coma cluster (Lal 2020).

Finally, we point out that in the first $\sim 400 \mathrm{kpc}$ the emission of the head-tail radio galaxy appears wobbly in the LOFAR high-resolution image, similarly to the cases of IC 711 and NGC 4869 (e.g., Sebastian et al. 2017; Wilber et al. 2019; Srivastava \& Singal 2020; Lal 2020, for recent works). The widening and narrowing features indicate the development of instabilities along the tail, for example as a consequence of the turbulent wake generated behind the host galaxy while it moves at high speed in the ICM (e.g., Jones \& Owen 1979).

\section{Conclusions}

We present a joint X-ray and radio analysis of the central region of A1775 $(z=0.072)$ employing Chandra, LOFAR $144 \mathrm{MHz}$, GMRT $235 \mathrm{MHz}$ and $610 \mathrm{MHz}$, and VLA $1.4 \mathrm{GHz}$ observations. Our main findings are summarized as follows.

1. We find clear evidence of gas motions in the cluster center and highlighted the presence of a cool, spiral-like structure in the thermal ICM. This pattern is observed in the X-ray surface brightness and in the temperature and pseudo-entropy maps.

2. At the NE boundary of the gas spiral, the X-ray surface brightness drops. The broken power-law fit indicates that the gas density changes by a factor $C \simeq 1.7$ across the jump. The inner and outer temperatures are $k T_{\text {in }}=3.0_{-0.4}^{+0.3} \mathrm{keV}$ and $k T_{\text {out }}=3.9_{-0.9}^{+1.5} \mathrm{keV}$ respectively, suggesting that the edge is tracing a cold front. 
3. We proposed two scenarios to explain the origin of the arcshaped feature revealed by Chandra. We argued that the preferred scenario is that of an off-axis merger along the line of sight, where the infalling subcluster has passed the pericenter and is turning around, producing a slingshot tail of low-entropy and low-temperature gas. Follow-up work is required to confirm the suggested dynamical configuration of the system.

4. Owing to the low-frequency observations performed with LOFAR and GMRT, we detected a $\sim 400 \mathrm{kpc}$ extension of the head-tail radio galaxy in A1775, leading to a total (projected) extent of this source of $\sim 800 \mathrm{kpc}$, doubling its previously reported size. The structure of the tail is collimated for the first $\sim 400 \mathrm{kpc}$, then it breaks and becomes more diffuse. This transition occurs at the location of the arc-shaped cold front. We speculate that the outer region of the tail originates from the re-acceleration of dormant tail electrons; deeper observations will shed light on this point.

5. At low frequencies, filamentary and diffuse radio emission is detected in the cluster center. The emission is recovered well only by LOFAR and is present also in the GMRT $235 \mathrm{MHz}$ data, suggesting an ultra-steep spectrum of $\alpha>2$. Due to its spectral properties, morphology, lack of clear optical counterpart(s) and possible connection with compression regions in the ICM, we classified it as revived fossil plasma.

6. We discovered extended, diffuse emission in the cluster center. This claim is solely based on the LOFAR low-resolution image. The source is bounded by the arc-shaped cold front. Under the assumption of a circular exponential model, the derived flux density at $144 \mathrm{MHz}$ is $S_{144}=245 \pm 50 \mathrm{mJy}$, corresponding to a radio power of $P_{144}=(3.1 \pm 0.7) \times$ $10^{24} \mathrm{~W} \mathrm{~Hz}^{-1}$. In line with the proposed dynamical scenario, we speculated that this central diffuse radio emission is tracing a slingshot radio halo. An intriguing indication of significant turbulent motions in the central region of the cluster is provided by the distortions observed in the filamentary structures F1 and F2.

Overall, our work indicates the presence of substantial dynamical gas motions in the central region of A1775. The discovery of a broken head-tail, revived fossil plasma, and a diffuse central emission probes ongoing interplay between thermal and nonthermal components in the ICM and particle reacceleration mechanisms in the cluster center.

Acknowledgements. We thank the anonymous referee for the useful comments on the manuscript. $\mathrm{AB}$ and $\mathrm{RJvW}$ acknowledge support from the VIDI research programme with project number 639.042 .729 , which is financed by the Netherlands Organisation for Scientific Research (NWO). FG, GB, RC, and MR acknowledge support from INAF mainstream project 'Galaxy Clusters Science with LOFAR' 1.05.01.86.05. VC acknowledges support from the Alexander von Humboldt Foundation. AD acknowledges support by the BMBF Verbundforschung under the grant 05A17STA. LOFAR (van Haarlem et al. 2013) is the LOw Frequency ARray designed and constructed by ASTRON. It has observing, data processing, and data storage facilities in several countries, which are owned by various parties (each with their own funding sources), and are collectively operated by the ILT foundation under a joint scientific policy. The ILT resources have benefitted from the following recent major funding sources: CNRS-INSU, Observatoire de Paris and Université d'Orléans, France; BMBF, MIWF-NRW, MPG, Germany; Science Foundation Ireland (SFI), Department of Business, Enterprise and Innovation (DBEI), Ireland; NWO, The Netherlands; The Science and Technology Facilities Council, UK; Ministry of Science and Higher Education, Poland; Istituto Nazionale di Astrofisica (INAF), Italy. This research made use of the Dutch national e-infrastructure with support of the SURF Cooperative (e-infra 180169) and the LOFAR e-infra group, and of the LOFAR IT computing infrastructure supported and operated by INAF, and by the Physics Dept. of Turin University (under the agreement with Consorzio Interuniversitario per la Fisica Spaziale) at the C3S Supercomputing Centre, Italy. The Jülich
LOFAR Long Term Archive and the German LOFAR network are both coordinated and operated by the Jülich Supercomputing Centre (JSC), and computing resources on the supercomputer JUWELS at JSC were provided by the Gauss Centre for Supercomputing e.V. (grant CHTB00) through the John von Neumann Institute for Computing (NIC). This research made use of the University of Hertfordshire high-performance computing facility and the LOFAR-UK computing facility located at the University of Hertfordshire and supported by STFC [ST/P000096/1]. We thank the staff of the GMRT for making these observations possible. GMRT is run by the National Centre for Radio Astrophysics of the Tata Institute of Fundamental Research. The NRAO is a facility of the National Science Foundation operated under cooperative agreement by Associated Universities, Inc. SRON is supported financially by NWO, The Netherlands Organisation for Scientific Research. Basic research in radio astronomy at the Naval Research Laboratory is supported by 6.1 Base funding. The scientific results reported in this article are based in part on data obtained from the Chandra Data Archive. This research made use of APLpy, an open-source plotting package for Python (Robitaille \& Bressert 2012).

Note added in proof. After this paper was accepted, Hu et al. (2021) made available results based on the analysis of Chandra and XMM-Newton observations of Abell 1775. Their findings are in good agreement with those presented in this paper. They also used numerical simulations and suggested that the X-ray properties observed in Abell 1775 could be produced by what we refer to as a sloshing scenario.

\section{References}

Andersson, K., Peterson, J., Madejski, G., \& Goobar, A. 2009, ApJ, 696, 1029 Andrade-Santos, F., Jones, C., Forman, W. R., et al. 2017, ApJ, 843, 76

Arnaud, K. 1996, in Astronomical Data Analysis Software and Systems V, eds. G. Jacoby, \& J. Barnes, ASP Conf. Ser., 101, 17

Ascasibar, Y., \& Markevitch, M. 2006, ApJ, 650, 102

Asplund, M., Grevesse, N., Sauval, A., \& Scott, P. 2009, ARA\&A, 47, 481

Bicknell, G. 1984, ApJ, 286, 68

Blanton, E. L., Randall, S. W., Clarke, T. E., et al. 2011, ApJ, 737, 99

Botteon, A., Gastaldello, F., \& Brunetti, G. 2018, MNRAS, 476, 5591

Botteon, A., Cassano, R., Eckert, D., et al. 2019, A\&A, 630, A77

Botteon, A., Brunetti, G., van Weeren, R. J., et al. 2020, ApJ, 897, 93

Boxelaar, J., van Weeren, R. J., \& Botteon, A. 2021, Astron. Comput., 35, 100464

Briggs, D. 1995, in American Astronomical Society Meeting Abstracts, Bull. Am. Astron. Soc., 27, 1444

Brunetti, G., \& Jones, T. W. 2014, IJMPD, 23, 30007

Brunetti, G., \& Lazarian, A. 2016, MNRAS, 458, 2584

Brunetti, G., \& Vazza, F. 2020, Phys. Rev. Lett., 124, 51101

Cassano, R., Botteon, A., Di Gennaro, G., et al. 2019, ApJ, 881, L18

Cavagnolo, K. W., Donahue, M., Voit, G. M., \& Sun, M. 2009, ApJS, 182, 12

Cavaliere, A., \& Fusco-Femiano, R. 1976, A\&A, 49, 137

Chambers, K., Magnier, E., Metcalfe, N., et al. 2016, ArXiv e-prints [arXiv:1612.05560]

Chandra, P., Ray, A., \& Bhatnagar, S. 2004, ApJ, 612, 974

Chen, H., Jones, C., Andrade-Santos, F., ZuHone, J. A., \& Li, Z. 2017, ApJ, 838, 38

Chincarini, G., Rood, H. J., Sastry, G. N., \& Welch, G. A. 1971, ApJ, 168, 11

Chow-Martínez, M., Andernach, H., Caretta, C., \& Trejo-Alonso, J. 2014, MNRAS, 445, 4073

Churazov, E., Forman, W. R., Jones, C., \& Böhringer, H. 2003, ApJ, 590, 225

Clarke, T. E., Blanton, E. L., \& Sarazin, C. L. 2004, ApJ, 616, 178

Cohen, A., \& Clarke, T. E. 2011, AJ, 141, 149

Cuciti, V., Brunetti, G., van Weeren, R. J., et al. 2018, A\&A, 609, A61

Davoust, E., \& Considere, S. 1995, A\&AS, 110, 19

de Gasperin, F., Intema, H. T., Shimwell, T. W., et al. 2017, Science Adv., 3 , e1701634

de Gasperin, F., Dijkema, T. J., Drabent, A., et al. 2019, A\&A, 622, A5

Douglass, E., Blanton, E. L., Randall, S. W., et al. 2018, ApJ, 868, 121

Dupke, R. A., \& Bregman, J. N. 2001, ApJ, 562, 266

Dupke, R. A., \& Bregman, J. N. 2006, ApJ, 639, 781

Dupke, R. A., Mirabal, N., Bregman, J. N., \& Evrard, A. E. 2007, ApJ, 668, 781

Ebeling, H., Edge, A., Böhringer, H., et al. 1998, MNRAS, 301, 881

Eckert, D., Molendi, S., \& Paltani, S. 2011, A\&A, 526, A79

Einasto, M., Tago, E., Jaaniste, J., Einasto, J., \& Andernach, H. 1997, A\&AS, 123,119

Enßlin, T. A., \& Krishna, G. 2001, A\&A, 366, 26 
Foreman-Mackey, D., Hogg, D. W., Lang, D., \& Goodman, J. 2013, PASP, 125, 306

Fujita, Y., Matsumoto, T., \& Wada, K. 2004, ApJ, 612, L9

Fujita, Y., Kohri, K., Yamazaki, R., \& Kino, M. 2007, ApJ, 663, L61

Gendron-Marsolais, M.-L., Hlavacek-Larrondo, J., van Weeren, R. J., et al. 2017 MNRAS, 469, 3872

Giacintucci, S., Venturi, T., Murgia, M., et al. 2007, A\&A, 476, 99

Giacintucci, S., Markevitch, M., Brunetti, G., et al. 2014a, ApJ, 795, 73

Giacintucci, S., Markevitch, M., Venturi, T., et al. 2014b, ApJ, 781, 9

Giacintucci, S., Markevitch, M., Cassano, R., et al. 2017, ApJ, 841, 71

Giacintucci, S., Markevitch, M., Cassano, R., et al. 2019, ApJ, 880, 70

Giovannini, G., \& Feretti, L. 2000, New Astron., 5, 335

Gitti, M., Brunetti, G., \& Setti, G. 2002, A\&A, 386, 456

Gunn, J., \& Gott, J. 1972, ApJ, 176, 1

Hallman, E. J., \& Markevitch, M. 2004, ApJ, 610, L81

Hardcastle, M. J., Gürkan, G., van Weeren, R. J., et al. 2016, MNRAS, 462, 1910

Hardcastle, M. J., Croston, J., Shimwell, T. W., et al. 2019, MNRAS, 488, 3416 Hintzen, P. 1979, PASP, 91, 426

Hitomi Collaboration (Aharonian, F., et al.) 2016, Nature, 535, 117

Hlavacek-Larrondo, J., Allen, S. W., Taylor, G. B., et al. 2013, ApJ, 777, 163

Hoang, D. N., Shimwell, T. W., Osinga, E., et al. 2021, MNRAS, 501, 576

Hu, D., Xu, H., Zhu, Z., et al. 2021, ApJ, submitted [arXiv:2103.03382]

Jenner, D. 1974, ApJ, 191, 55

Johnson, R. E., Markevitch, M., Wegner, G., Jones, C., \& Forman, W. R. 2010, ApJ, 710, 1776

Johnson, R. E., ZuHone, J. A., Jones, C., Forman, W. R., \& Markevitch, M. 2012 ApJ, 751, 95

Jones, T. W., \& Owen, F. N. 1979, ApJ, 234, 818

Kale, R., \& Dwarakanath, K. 2012, ApJ, 744, 46

Keshet, U., \& Loeb, A. 2010, ApJ, 722, 737

Kirshner, R., Oemler, A., Jr., Schechter, P., \& Shectman, S. 1983, AJ, 88, 1285

Kopylov, A., \& Kopylova, F. 2009, Astrophys. Bull., 64, 207

Laganá, T., Andrade-Santos, F., \& Lima Neto, G. 2010, A\&A, 511, A15

Laganá, T., Durret, F., \& Lopes, P. A. 2019, MNRAS, 484, 2807

Laing, R., \& Bridle, A. 2002a, MNRAS, 336, 1161

Laing, R., \& Bridle, A. 2002b, MNRAS, 336, 328

Lal, D. V. 2020, AJ, 160, 161

Liu, A., Yu, H., Tozzi, P., \& Zhu, Z.-H. 2015, ApJ, 809, 27

Liu, A., Yu, H., Tozzi, P., \& Zhu, Z.-H. 2016, ApJ, 821, 29

Loken, C., Roettiger, K., Burns, J. O., \& Norman, M. 1995, ApJ, 445, 80

Lopes, P. A., Trevisan, M., Laganá, T., et al. 2018, MNRAS, 478, 5473

Mandal, S., Intema, H. T., van Weeren, R. J., et al. 2020, A\&A, 634, A4

Markevitch, M., \& Vikhlinin, A. 2007, Phys. Rep., 443, 1

Maughan, B. J., Giles, P., Randall, S. W., Jones, C., \& Forman, W. R. 2012 MNRAS, 421, 1583

Mazzotta, P., \& Giacintucci, S. 2008, ApJ, 675, L9

Murgia, M., Govoni, F., Markevitch, M., et al. 2009, A\&A, 499, 679

Nandra, K., Barret, D., Barcons, X., et al. 2013, ArXiv e-prints [arXiv:1306.2307]

O’Donoghue, A. A., Eilek, J. A., \& Owen, F. N. 1993, ApJ, 408, 428

Oegerle, W. R., Hill, J. M., \& Fitchett, M. J. 1995, AJ, 110, 32

Offringa, A. R., \& Smirnov, O. M. 2017, MNRAS, 471, 301

Offringa, A. R., McKinley, B., Hurley-Walker, N., et al. 2014, MNRAS, 444, 606

Osinga, E., van Weeren, R. J., Boxelaar, J., et al. 2021, A\&A, 648, A11

Owen, F. N., \& Ledlow, M. J. 1997, ApJS, 108, 41

Owers, M. S., Nulsen, P. E., Couch, W., et al. 2014, ApJ, 780, 163

Parma, P., de Ruiter, H., \& Cameron, R. 1991, AJ, 102, 1960

Perley, R. A., \& Butler, B. 2017, ApJS, 230, 7
Pfrommer, C., \& Enßlin, T. A. 2004, A\&A, 413, 17 Planck Collaboration XXVII 2016, A\&A, 594, A27

Poole, G., Fardal, M., Babul, A., et al. 2006, MNRAS, 373, 881

Reiss, I., \& Keshet, U. 2014, Phys. Rev. Lett., 113, 71302

Robitaille, T., \& Bressert, E. 2012, Astrophysics Source Code Library [record ascl:1208.017]

Roediger, E., Brüggen, M., Simionescu, A., et al. 2011, MNRAS, 413, 2057

Roediger, E., Lovisari, L., Dupke, R. A., et al. 2012, MNRAS, 420, 3632

Roediger, E., Kraft, R. P., Nulsen, P. E., et al. 2015, ApJ, 806, 104

Rossetti, M., Eckert, D., De Grandi, S., et al. 2013, A\&A, 556, A44

Sanders, J. S. 2006, MNRAS, 371, 829

Sanders, J. S., Fabian, A. C., Taylor, G. B., et al. 2016a, MNRAS, 457, 82

Sanders, J. S., Fabian, A. C., Russell, H., Walker, S., \& Blundell, K. M. 2016b, MNRAS, 460, 1898

Sanders, J. S., Dennerl, K., Russell, H., et al. 2020, A\&A, 633, A42

Santos, J., Rosati, P., Tozzi, P., et al. 2008, A\&A, 483, 35

Sarazin, C. L. 1986, Rev. Mod. Phys., 58, 1

Sebastian, B., Lal, D. V., \& Pramesh Rao, A. 2017, AJ, 154, 169

Sheardown, A., Fish, T. M., Roediger, E., et al. 2019, ApJ, 874, 112

Shimwell, T. W., Röttgering, H. J., Best, P. N., et al. 2017, A\&A, 598, A104

Shimwell, T. W., Tasse, C., Hardcastle, M. J., et al. 2019, A\&A, 622, A1

Slee, O., Roy, A., Murgia, M., Andernach, H., \& Ehle, M. 2001, AJ, 122, 1172

Smirnov, O. M., \& Tasse, C. 2015, MNRAS, 449, 2668

Srivastava, S., \& Singal, A. K. 2020, MNRAS, 493, 3811

Tasse, C. 2014a, ArXiv e-prints [arXiv:1410.8706]

Tasse, C. 2014b, A\&A, 566, A127

Tasse, C., Hugo, B., Mirmont, M., et al. 2018, A\&A, 611, A87

Tasse, C., Shimwell, T. W., Hardcastle, M. J., et al. 2021, A\&A, 648, A1

Terni de Gregory, B., Feretti, L., Giovannini, G., et al. 2017, A\&A, 608, A58

Tittley, E. R., \& Henriksen, M. 2005, ApJ, 618, 227

Ueda, S., Kitayama, T., \& Dotani, T. 2017, ApJ, 837, 34

van Haarlem, M., Wise, M. W., Gunst, A., et al. 2013, A\&A, 556, A2

van Weeren, R. J., Röttgering, H. J., \& Brüggen, M. 2011, A\&A, 527, A114

van Weeren, R. J., Williams, W. L., Hardcastle, M. J., et al. 2016, ApJS, 223, 2

van Weeren, R. J., Andrade-Santos, F., Dawson, W. A., et al. 2017, Nature Astron., 1, 5

van Weeren, R. J., de Gasperin, F., Akamatsu, H., et al. 2019, Space Sci. Rev., 215,16

van Weeren, R. J., Shimwell, T. W., Botteon, A., et al. 2020, A\&A, submitted, ArXiv e-prints [arXiv:2011.02387]

Venturi, T., Rossetti, M., Bardelli, S., et al. 2013, A\&A, 558, A146

Wilber, A. G., Brüggen, M., Bonafede, A., et al. 2018, MNRAS, 473, 3536

Wilber, A. G., Brüggen, M., Bonafede, A., et al. 2019, A\&A, 622, A25

Williams, W. L., van Weeren, R. J., Röttgering, H. J., et al. 2016, MNRAS, 460 2385

Willingale, R., Starling, R., Beardmore, A., Tanvir, N., \& O’Brien, P. 2013, MNRAS, 431, 394

XRISM Science Team 2020, ArXiv e-prints [arXiv:2003.04962]

Zabludoff, A. I., Huchra, J. P., \& Geller, M. J. 1990, ApJS, 74, 1

Zandanel, F., Pfrommer, C., \& Prada, F. 2014, MNRAS, 438, 124

Zhang, L., Yuan, Q., Yang, Q., et al. 2011, PASJ, 63, 585

ZuHone, J. A., \& Roediger, E. 2016, J. Plasma Phys., 82, 48

ZuHone, J. A., Markevitch, M., \& Johnson, R. E. 2010, ApJ, 717, 908

ZuHone, J. A., Markevitch, M., \& Lee, D. 2011, ApJ, 743, 16

ZuHone, J. A., Markevitch, M., Brunetti, G., \& Giacintucci, S. 2013, ApJ, 762, 78

ZuHone, J. A., Brunetti, G., Giacintucci, S., \& Markevitch, M. 2015, ApJ, 801, 146 


\section{Appendix A: Spherical vs. elliptical $\beta$-models}

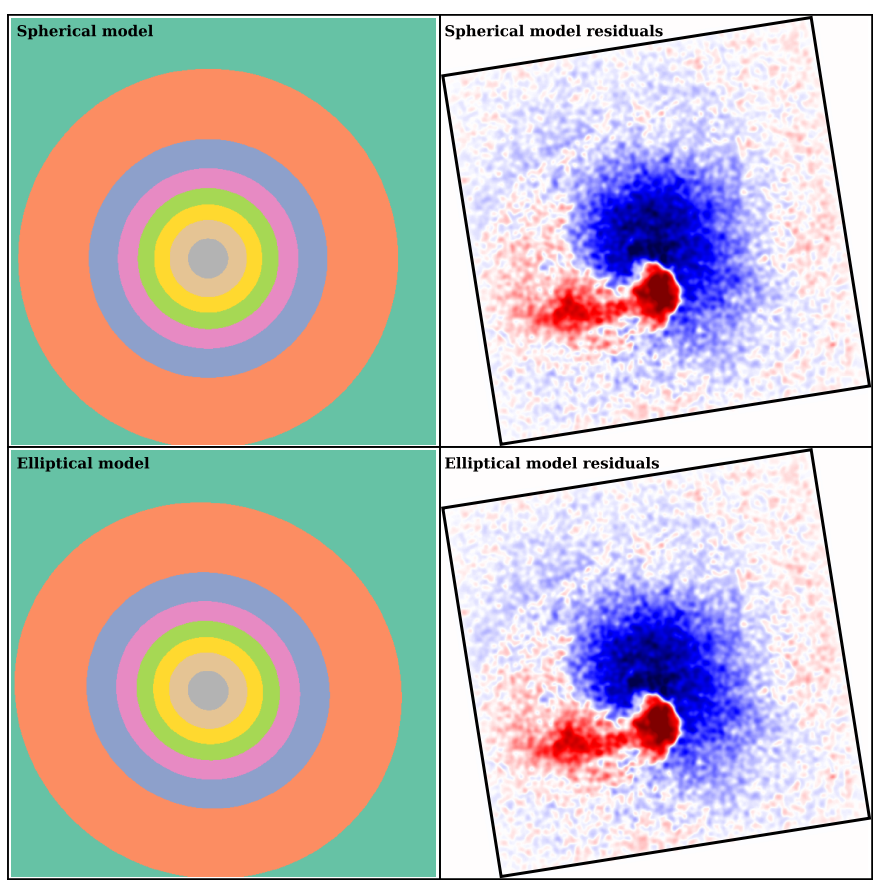

Fig. A.1. Visual comparison between best-fit spherical and elliptical $\beta$ models (left panels) and their residuals (right panels).

By using the CIAO task dmellipse, we found that an ellipse that encloses $90 \%$ of the ICM photons has a ratio between major and minor axis of 1.05 and orientation angle of $333^{\circ}$. Due to the small axis ratio, an elliptical $\beta$-model with this shape does not provide any significant difference with respect to the spherical model adopted in the main text, and shows the same spiral-like pattern in the residual image (Fig. A.1). The best-fit elliptical model has a core radius of $r_{\mathrm{c}}=2.63 \pm 0.05$ arcmin and $\beta=0.84 \pm$ 0.01 , which are consistent with the values reported in Sect. 3.1 for the spherical model.

\section{Appendix B: Error maps and corner plot}

The temperature error map is reported in Fig. B.1. The pseudopressure and pseudo-entropy maps have similar fractional errors given the small uncertainty (a few percent) associated to the emission measure from which they depend (see Botteon et al. 2018). The low- and high-frequency spectral index error maps are reported in Fig. B.2.

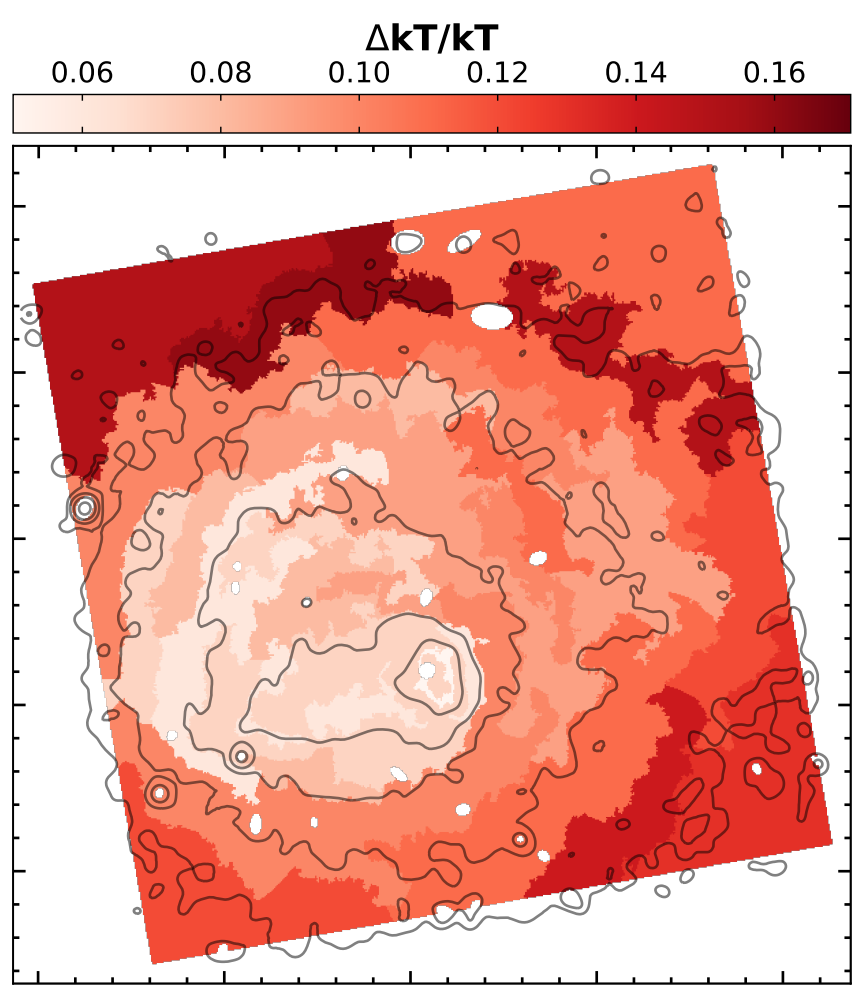

Fig. B.1. Temperature error map corresponding to Fig. 3.
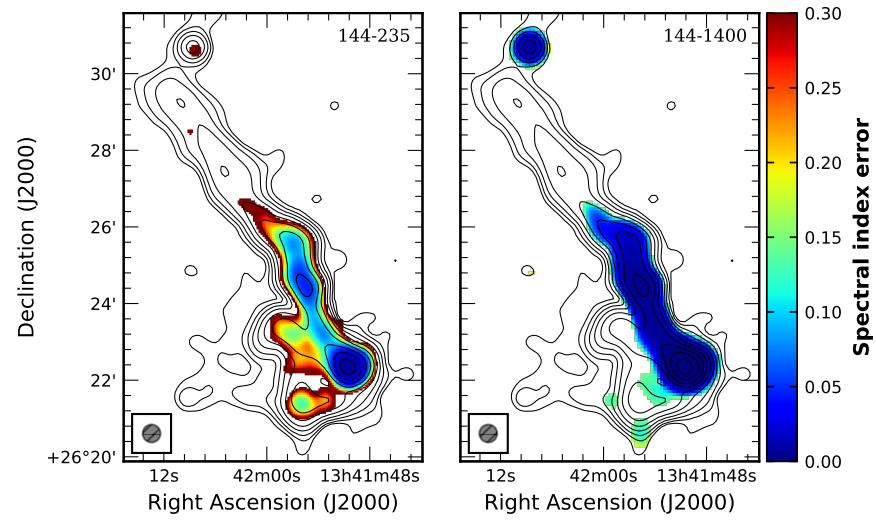

Fig. B.2. Spectral index error maps corresponding to Fig. 9.

The MCMC corner plot (Foreman-Mackey et al. 2013) showing the distribution of solutions for the fit parameters of the central diffuse emission surface-brightness profile is shown in Fig. B.3. 
A. Botteon et al.: Nonthermal phenomena in the center of Abell 1775

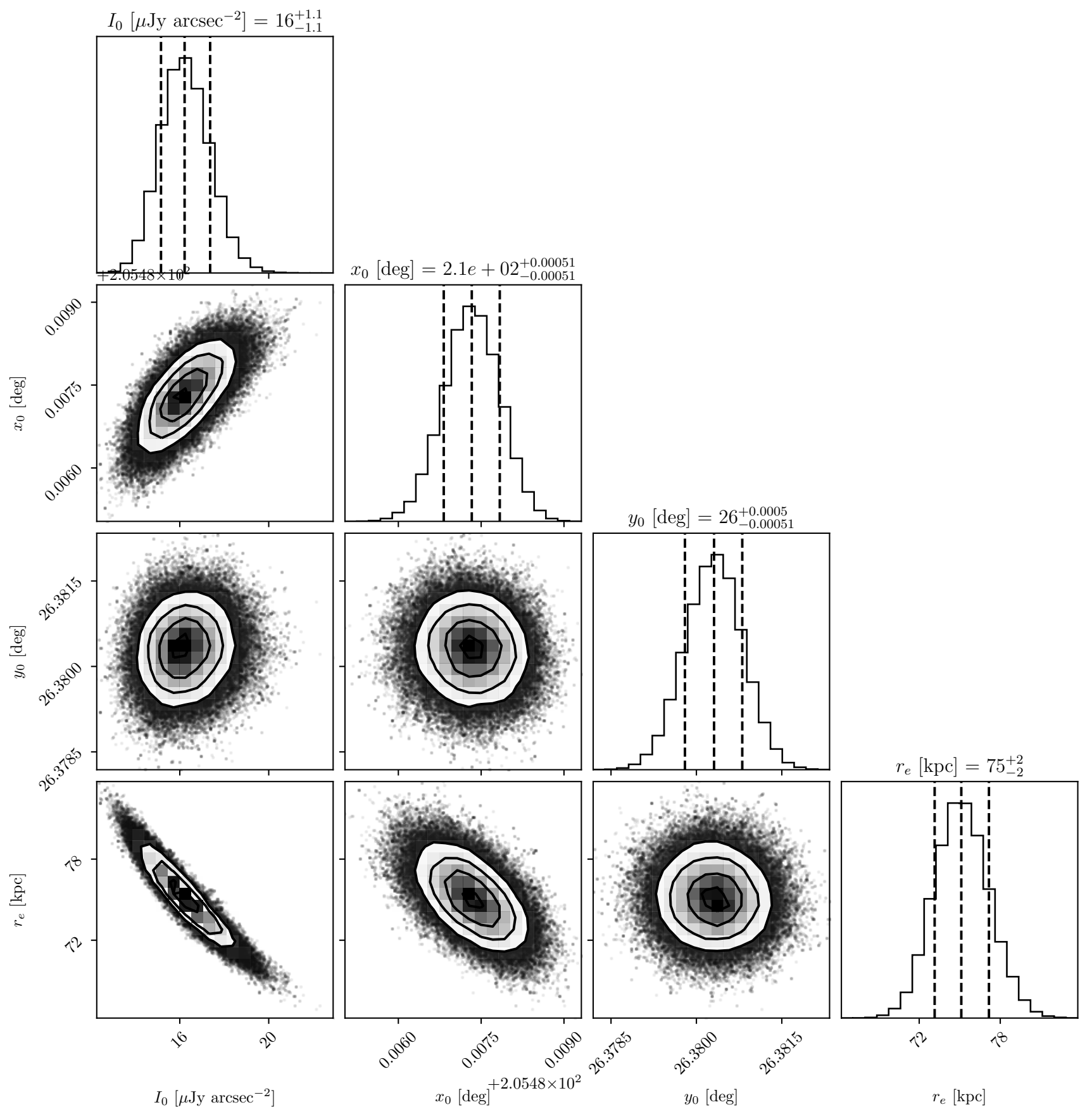

Fig. B.3. MCMC corner plot for the central diffuse emission surface-brightness profile fit corresponding to Fig. 11. 